\title{
LA-UR- 09002599
}

Approved for public release;

distribution is unlimited.
Title:

Author(s):

Intended for:
Peristaltic Particle Transport using the Lattice Boltzmann Method

\section{Kevin Connington \\ Qinjun Kang \\ Hari Viswanathan \\ Shiyi Chen \\ Amr Abdel-Fattah}

\section{Publication in journal: Physics of Fluids}

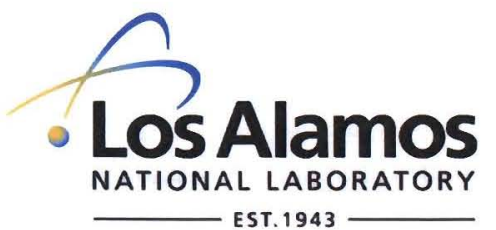

Los Alamos National Laboratory, an affirmative action/equal opportunity employer, is operated by the Los Alamos National Security, LLC for the National Nuclear Security Administration of the U.S. Department of Energy under contract DE-AC52-06NA25396. By acceptance of this article, the publisher recognizes that the U.S. Government retains a nonexclusive, royalty-free license to publish or reproduce the published form of this contribution, or to allow others to do so, for U.S. Government purposes. Los Alamos National Laboratory requests that the publisher identify this article as work performed under the auspices of the U.S. Department of Energy. Los Alamos National Laboratory strongly supports academic freedom and a researcher's right to publish; as an institution, however, the Laboratory does not endorse the viewpoint of a publication or guarantee its technical correctness. 


\title{
Peristaltic Particle Transport using the Lattice Boltzmann Method
}

\author{
Kevin Connington, ${ }^{1, *}$ Qinjun Kang, ${ }^{2}$ Hari Viswanathan, ${ }^{2}$ \\ Amr Abdel-Fattah, ${ }^{2}$ and Shiyi Chen ${ }^{1,3}$ \\ ${ }^{1}$ Department of Mechanical Engineering, The Johns Hopkins University, Baltimore, MD 21218, USA \\ ${ }^{2}$ Earth and Environmental Sciences Division, Los Alamos National Laboratory, Los Alamos, NM 87545, USA \\ ${ }^{3}$ CoE and CCSE, Peking University, Beijing, China \\ Submitted to Physics of Fluids: October 9, 2008
}

\begin{abstract}
Peristaltic transport refers to a class of internal fluid flows where the periodic deformation of flexible containing walls elicits a non-negligible fluid motion. It is a mechanism used to transport fluid and immersed solid particles in a tube or channel when it is ineffective or impossible to impose a favorable pressure gradient or desirous to avoid contact between the transported mixture and mechanical moving parts. Peristaltic transport occurs in many physiological situations and has myriad industrial applications. We focus our study on the peristaltic transport of a macroscopic particle in a two dimensional channel using the Lattice Boltzmann Method(LBM). We systematically investigate the effect of variation of the relevant dimensionless parameters of the system on the particle transport. We find, among other results, a case where an increase of Reynolds number can actually lead to a slight increase in particle transport, and a case where as the wall deformation
\end{abstract}

*Author to whom correspondence should be addressed. Electronic mail: kconnin1@jhu.edu 
increases, the motion of the particle becomes non-negative only. We examine the particle behavior when the system exhibits the peculiar phenomenon of fluid trapping. Under these circumstances, the particle may itself become trapped where it is subsequently transported at the wave speed, which is the maximum possible transport in the absence of a favorable pressure gradient. Finally, we analyze how the particle presence affects stress, pressure, and dissipation in the fluid in hopes of determining preferred working conditions for peristaltic transport of shear-sensitive particles. We find that the levels of shear stress are most hazardous near the throat of the channel. We advise that shear-sensitive particles should be transported under conditions where trapping occurs as the particle is typically situated in a region of innocuous shear stress levels.

\section{Introduction}

A peristaltic flow occurs when a tube or channel with flexible walls transports the contained fluid by progressing a series of contraction or expansion waves along the length of those walls. The topic of this discourse is the peristaltic transport of a macroscopic solid particle immersed in the carrier fluid.

Peristaltic transport is a process that occurs frequently both in nature and industrial applications. Interest in the study of peristaltic flows was sparked by physiological concerns $[1,2,3,4]$. For example, peristaltic transport occurs in the ureter, the gastrointestinal tract, and in the vasomotion of blood vessels. In an industrial context, peristalsis may be useful when it is necessary to transport fluid while avoiding direct contact with mechanical moving parts. Hanin [5] suggested, "This need may arise when the fluid is very corrosive or toxic, ... or when the fluid carries solid particles for which a passage free of obstacles would be desireable." Industrial applications of peristaltic transport include 
mining processes involving corrosive cyanide for gold recovery and biomedical devices to circulate blood between a patient and a heart-lung machine during cardiovasular surgery. Examples of peristaltic pumping of solid particles include egg transport in Fallopian tubes, food bolus transport in the gastro-intestinal tract, and calculus transport in the ureter. Many cases of peristalsis operate under working conditions of complete occlusion, where the distensible walls converge to produce zero lumen. This is the most efficient implementation of peristalsis, as the compartmentalized fluid is transported through the tube by positive displacement of the walls at the wave speed. However, peristaltic transport at moderate compression can still be an efficacious process.

The early analytical work on the subject was instrumental in establishing a sense of the typical qualitative nature of peristaltic flows $[1,2,6,7,8]$. Cognizant that they were not mimicing physiological systems exactly [9], these workers derived many useful results by analyzing idealized situations, designating one or more physical parameters as small or negligible to facilitate their study.

The following are some highlights of peristaltic pumping that were succinctly summarized by Jaffrin \& Shapiro [10]. The contraction of the walls produces a rising pressure gradient in the direction of the wave. Peristalsis is an inherently viscous process, a requisite condition for the pressure gradient to occur. In light of this observation, most of the analytical work was performed in the limit of small or negligible inertia, though some authors did not employ this restriction. In this limit, the instantaneous velocity is reminiscent of Poiseuille flow over a cross section. When the wavelength of the deformation is large compared to the width of the channel, the curvature of the flow streamlines is small, and the pressure can be considered uniform over the cross section. The flow is aligned with the wave in dilated sections of the channel, while it is oriented opposite to the wave in contracted sections. Though the flow changes direction as the wave progresses, the 
volume flow rate averaged over a wave period is in the same direction as the wave propogation, unless an overwhelming adverse pressure gradient is imposed on the system. These results were obtained for an infinite train of periodic waves, where it was proven sufficient to analyze a single wave with periodic inlet and outlet boundaries.

Under certain circumstances, peristaltic flows have been seen to exhibit the peculiar behaviors of trapping and reflux [1]. Trapping occurs in cases where the deformation is large, and is characterized by a bolus of fluid which travels with the wave. It can only be observed analytically through streamlines calculated in a frame of reference moving with the wave, and is visualized as a pair of recirculating vortices. Since the streamlines are closed, the recirculating fluid must travel at the wave speed in the average sense. Over the years, a semantic debate took place over the meaning attributed to the term reflux. The dichotomy split those involved into two factions, one defining reflux to be the reverse transport of fluid in the averaged Eulerian sense $[2,7]$, and the other labeling it as the reverse transport of Lagrangian fluid particles [1].

These analytical results were corroborated by several experimental endeavors [11, 12]. Since no experiment can rigorously simulate an 'infinite wave train', Weinberg et al. [12] determined an experiment must possess three criteria to sufficiently approximate the situation: 1) the deformation wave must be periodic; 2) there must be an integral number of wavelengths between inlet and outlet resevoirs; and 3) the imposed pressure difference between the resevoirs must not change with time. These criteria are important to keep in mind as any numerical simulation should satisfy these conditions as well. Hung \& Brown [13] performed experiments of peristaltic transport of macroscopic particles. They investigated the particle transport produced from the passage of a single wave of deformation in an approximately two-dimensional channel. They studied the effects of particle size, channel width, particle shape, and Reynolds number. There have been 
numerous numerical studies of peristaltic transport $[14 ; 15,16]$, where the restrictions on various parameters are less prohibitive than in theoretical studies. Recently there have been numerical studies of closed cavity peristalsis $[17,18]$ and solid particle suspensions $[19,20,21]$, as well as innovative theoretical studies [22]. To the best of the authors' knowledge, the work of Fauci [23], utilizing the Immersed Boundary Method (IBM), was the only previous numerical instance of peristaltic transport of a finite-sized particle.

In this paper, we will use the Lattice Boltzmann Method (LBM) to perform simulations of the peristaltic transport of a macroscopic particle. We systematically study how the transport is affected by varying each of the dimensionless parameters of the system independently. We also discover that the periodic boundary conditions can substantially affect the particle transport more than anticipated in certain circumstances. We examine the particle behavior when introduced to a system that exhibits trapping, and discover that the particle can enter and remain inside the trapped fluid bolus. This allows the particle to be transported at the wave speed. We analyze the effect of the particle on stress, pressure, and dissipation in the fluid, and find that the particle enhances these quantities near the throat of the channel while having very little influence in the dilated section.

To perform these simulations more efficiently, we have parallelized the code using MPI(Message Passing Interface) while augmenting the standard LBM in several respects. We removed the fluid from nodes that are considered interior to solid boundaries as suggested by Aidun [24]; adopted the method of Yu et al. [25] to achieve second-order accuracy on the solid boundary conditions; and implemented the Momentum Exchange (ME) scheme to calculate the force exerted by the fluid on solid boundaries as developed by Ladd [26]. The numerical method is elobarated upon in Section 2. The problem specification is laid out in detail in Section 3. The code is validated in Section 4 through 


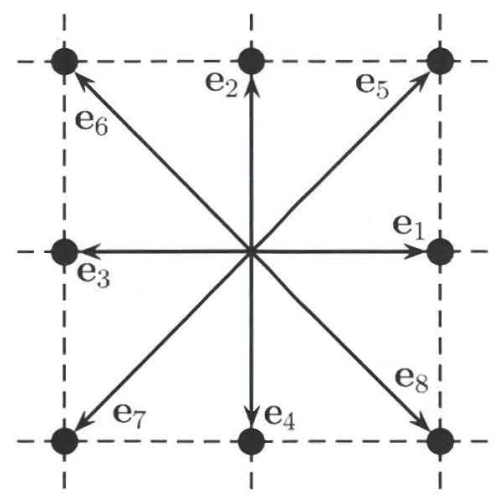

Figure 1: Lattice structure of the two-dimensional "D2Q9" lattice.

comparisons with particle-free analytical solutions and the particle-laden simulations of Fauci. The results are presented in Sections 5 and 6. Finally some concluding remarks are discussed in Section 7.

\section{Numerical Method}

\subsection{Lattice Boltzmann Model}

The Lattice Boltzmann Equation (LBE) [27, 28, 29, 30] with single relaxation time (SRT) approximation $[31,32]$ is written:

$$
f_{\alpha}\left(\boldsymbol{x}+\boldsymbol{e}_{\alpha} \delta t, t+\delta t\right)=f_{\alpha}(\boldsymbol{x}, t)-\frac{1}{\tau}\left[f_{\alpha}(\boldsymbol{x}, t)-f_{\alpha}^{(e q)}(\boldsymbol{x}, t)\right]
$$

where $\tau$ is the relaxation time, $\boldsymbol{x}$ is a discretized point in physical space, $t$ is the discretized time, and $\delta t$ is the time step. $f_{\alpha}$ and $f_{\alpha}^{(e q)}$ are the distribution function and corresponding equilibrium distribution function associated with the $\alpha$ th discrete velocity direction $\boldsymbol{e}_{\alpha}$, respectively. In the two-dimensional nine-velocity lattice (D2Q9) model shown in Fig. 1, the discrete velocity set is: 


$$
\boldsymbol{e}_{\alpha}= \begin{cases}(0,0) & \text { for } \alpha=0 \\ c(\cos ((\alpha-1) \pi / 2), \sin ((\alpha-1) \pi / 2)) & \text { for } \alpha=1-4 \\ \sqrt{2} c(\cos ((2 \alpha-1) \pi / 4), \sin ((2 \alpha-1) \pi / 4)) & \text { for } \alpha=5-8 .\end{cases}
$$

Here the lattice speed $c=\delta x / \delta t$, where $\delta x$ is the lattice constant; both $\delta x$ and $\delta t$ are set to unity. The equilibrium distribution functions for this lattice are of the form:

$$
f_{\alpha}^{(e q)}=\rho w_{\alpha}\left[1+\frac{3}{c^{2}} \boldsymbol{e}_{\alpha} \cdot \boldsymbol{u}+\frac{9}{2 c^{4}}\left(\boldsymbol{e}_{\alpha} \cdot \boldsymbol{u}\right)^{2}-\frac{3}{2 c^{2}} u^{2}\right]
$$

where $w_{\alpha}$ is the weighting factor, $\rho$ is the fluid density, and $\boldsymbol{u}$ is the fluid velocity. The weighting factors for this model are:

$$
w_{\alpha}= \begin{cases}4 / 9 & \text { for } \alpha=0 \\ 1 / 9 & \text { for } \alpha=1-4 \\ 1 / 36 & \text { for } \alpha=5-8\end{cases}
$$

The macroscopic Navier-Stokes equations can be obtained from the LBE through a Chapman-Enskog expansion [33].

\subsection{Complex Boundary Conditions}

Consider the wall that is represented in Fig. 2. The particle is mapped onto the existing mesh and the boundary divides the solid boundary region $\boldsymbol{x}_{b}$, from the fluid region $\boldsymbol{x}_{f}$. In his simulations of suspension flows, Ladd $[26,34]$ placed the wall along the link, half-way between the fluid and boundary node, referred to as Bounce-Back along the Link (BBL). This unfortunately portrays a curved boundary as a series of steps, and under certain 


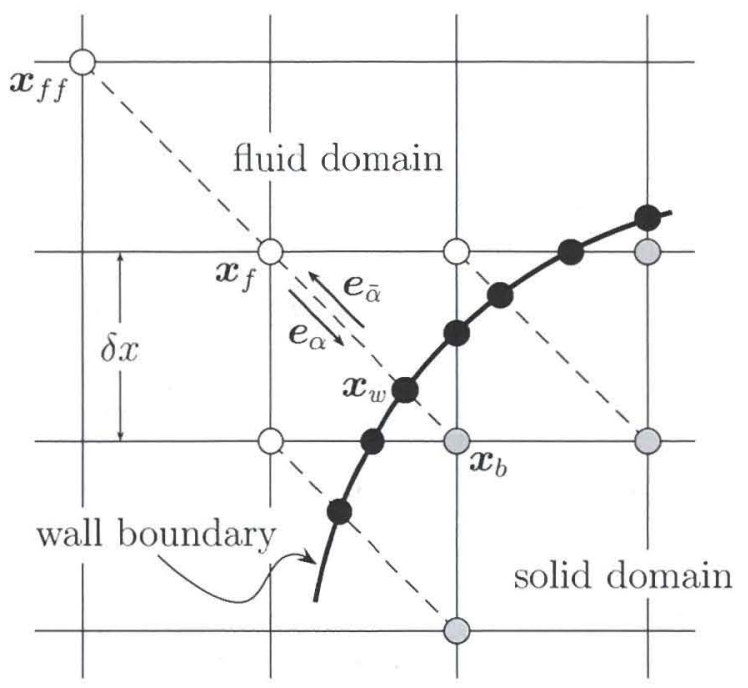

Figure 2: A curved wall solid boundary on a 2D lattice. $\boldsymbol{x}_{f}$ are fluid nodes, $\boldsymbol{x}_{b}$ are boundary nodes inside the solid, and $\boldsymbol{x}_{w}$ are the locations where the lattice vectors intersect the solid wall.

circumstances dubiously represents the actual geometry. To achieve the highest possible accuracy, the boundary conditions should account for the exact position of the wall $\left(\boldsymbol{x}_{w}\right)$ along the link. For simulations that involve moving complex boundaries, we employ the method of $\mathrm{Yu}$ et al. [25]; they have demonstrated their scheme to be both accurate and stable.

According to the terminology of Fig. 2, $\Delta$ represents the fraction of an intersected link inside the fluid region, and is given by:

$$
\Delta=\frac{\left|x_{f}-x_{w}\right|}{\left|x_{f}-x_{b}\right|}
$$

The boundary condition procedure is initiated after the streaming step. One first calculates the $\alpha$-direction distribution function at the position $\boldsymbol{x}_{w}$ along the intersected link using a suitable interpolation. The two-point interpolation scheme is 


$$
f_{\alpha}\left(\boldsymbol{x}_{w}, t+\delta t\right)=f_{\alpha}\left(\boldsymbol{x}_{f}, t+\delta t\right)+\Delta\left[f_{\alpha}\left(\boldsymbol{x}_{b}, t+\delta t\right)-f_{\alpha}\left(\boldsymbol{x}_{f}, t+\delta t\right)\right]
$$

The no-slip condition is then fulfilled on the wall by specifying the $\bar{\alpha}$-direction distribution function while taking the wall velocity into account:

$$
f_{\bar{\alpha}}\left(\boldsymbol{x}_{w}, t+\delta t\right)=f_{\alpha}\left(\boldsymbol{x}_{w}, t+\delta t\right)+2 w_{\alpha} \rho_{w} \frac{3}{c^{2}} \boldsymbol{e}_{\bar{\alpha}} \cdot \boldsymbol{u}_{w},
$$

where $\rho_{w}$ is the fluid density at the wall. Yu et al. specified $\rho_{w}=\rho\left(\boldsymbol{x}_{f}\right)$, since the flow is nearly incompressible. The procedure is completed by using another suitable interpolation to compute the desired distribution function:

$$
f_{\bar{\alpha}}\left(\boldsymbol{x}_{f}\right)=f_{\bar{\alpha}}\left(\boldsymbol{x}_{w}\right)+\frac{\Delta}{1+\Delta}\left[f_{\bar{\alpha}}\left(\boldsymbol{x}_{f}+\boldsymbol{e}_{\bar{\alpha}}\right)-f_{\bar{\alpha}}\left(\boldsymbol{x}_{w}\right)\right],
$$

where the equation is evaluated at time $t+\delta t$.

This method is an adaptation of the original second-order boundary condition proposed by Filippova and Hänel [35], and improved upon by Mei et al [36]. The advantage of $\mathrm{Yu}$ et al.'s scheme is that it utilizes a single interpolation regardless of the value of $\Delta$. The earlier schemes employed two distict equations to evaluate the interpolation, depending on whether $\Delta$ was above or below a threshold value.

Due to the movement of solid boundaries over the lattice, certain fluid nodes will be covered by the solid walls. The fluid from these nodes is removed from the system $[24,37]$. Conversely, a node originally in the solid region can be uncovered and become part of the fluid domain. When such a fluid node is recovered, the distribution function at this newly created node, $\boldsymbol{x}_{r}$, is assumed to be the average of the extrapolated distribution function values from a second order extrapolation scheme over all the possible directions [38]. A 
possible direction is defined to be any direction $\boldsymbol{e}_{\alpha}$ where both $\boldsymbol{x}_{r}+\boldsymbol{e}_{\alpha}$ and $\boldsymbol{x}_{r}+2 \boldsymbol{e}_{\alpha}$ are pre-existing fluid nodes; i.e. these nodes are niether solid nodes nor recovered nodes themselves. The extrapolation is stated

$$
f_{\alpha}\left(\boldsymbol{x}_{r}\right)=\frac{\sum_{j \in S}\left\{2 f_{\alpha}\left(\boldsymbol{x}_{r}+\boldsymbol{e}_{j}\right)-f_{\alpha}\left(\boldsymbol{x}_{r}+2 \boldsymbol{e}_{j}\right)\right\}}{N_{S}} \quad \forall \alpha,
$$

where $S$ is the set of all possible directions, and $N_{S}$ is the number of elements in $\mathrm{S}$. Fang et al. [39] has shown that the mass is approximately conserved at the boundaries.

\subsection{Force Calculation on Particle Surface}

Since the motion of an immersed solid particle and the carrier fluid are coupled, it is important to accurately calculate the hydrodynamic force and torque exerted on the particle by the fluid. The Momentum Exchange (ME) method [26, 34, 40], unique to the lattice Boltzmann method, is ubiquitous throughout many LBM simulations involving force calculation. The popularity of the method stems from its accuracy and ease of implementation in both two and three dimensions, facilitating one of the main advantages of LBM simulations in general.

For each link connecting a fluid node to a boundary node, the fluid exerts a force on the particle in that particular link direction, realized as an impulse of momentum exchanged between the fluid and the particle. According to the terminology of Fig. 2, the force exerted on the particle at $\boldsymbol{x}_{w}$ along a relevant direction $\boldsymbol{e}_{\alpha}$ connecting a fluid node at $\boldsymbol{x}_{f}$ to a boundary node at $\boldsymbol{x}_{b}$ is

$$
\boldsymbol{F}\left(\boldsymbol{x}_{w}, \boldsymbol{e}_{\alpha}\right)=\boldsymbol{e}_{\alpha}\left[f_{\alpha}\left(\boldsymbol{x}_{w}, t+\delta t\right)+f_{\bar{\alpha}}\left(\boldsymbol{x}_{w}, t+\delta t\right)\right],
$$


where $f_{\alpha}\left(\boldsymbol{x}_{w}, t+\delta t\right)$ and $f_{\bar{\alpha}}\left(\boldsymbol{x}_{w}, t+\delta t\right)$ are computed in equations (6) and (7). The total force on the particle is the sum of the forces computed from each relevant link connecting a fluid node to a boundary node. The total torque can also be computed from this information [41]. Mei et al. [40] concluded that a minimum resolution of ten lattice units across the diameter of a fixed circular particle was necessary to accurately calculate the drag at $R e \sim 100$; we have established a minumum resolution for this work double that which was suggested to lend additional credence to the integrity of our results. The translation and rotation of the particle are updated each Newtonian dynamics time step using a half-step "leap-frog" scheme [42, 43].

Due to the fact that the particle is free to move in response to the forces imposed on it, it was possible for it to stray very near, or even to contact the peristaltic walls. If the particle is situated near the wall such that there are no fluid nodes between the particle and the wall over some element of the particle surface, the total force on the particle would be miscalculated as the information necessary to apply the ME method would not be availible over that region. Moreover, the particle boundary should not overlap the wall surface. To resolve these issues, we incorporated the use of a short-range repulsive force applied to the particle at the location on the surface where the particle-wall gap is a minimum, imposed only during time steps when the minimum gap is below a threshold value $[44,45]$. Throughout the simulations performed in this paper however, the particle never migrates so close to the wall, and this algorhithm is not invoked.

\section{Problem Specification}

The geometric configuration and nomenclature for the problem are portrayed in Fig. 3. To avoid confusion, we have tried to remain consistent with the variable names and definitions 


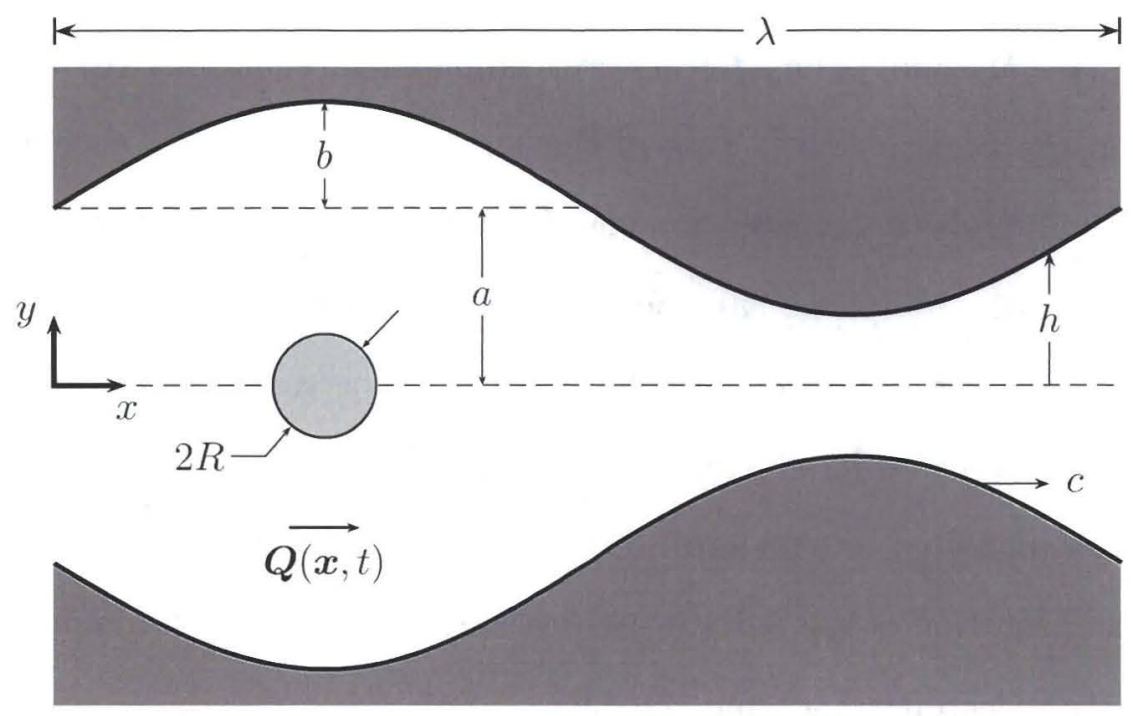

Figure 3: Geometry and nomenclature for peristaltic flow with a transport particle.

given by Shapiro et al [1]. A macroscopic circular particle, free to move in response to an applied force, is immersed in a fluid between flexible walls that are constrained to move in a prescribed manner, referred to hereafter as peristaltic walls. The imposed motion of the walls induces a fluid flow, which serves to transport the particle. While there is an abundance of phenomena worthy of study associated with peristaltic flows, we focus on this macroscopic particle transport.

Aside from the presence of the particle, the geometry of our simulation is commonly seen throughout the literature. The peristaltic walls are of a sinusoidal nature, symmetric about the centerline of the channel. A transverse wave of deformation alternately contracts and expands the wall position as it progresses in the longitudinal direction. The length of the domain is restricted to be an integral number of wavelengths of the periodic function defining the wall shape. The no-slip condition is enforced on the moving peristaltic walls and solid particle as detailed in Section 2.2, while the boundary conditions at the inlet and outlet are of a periodic type. 
According to Fig. 3, the mean half-width of the channel is $a$, and the amplitude of wall deformation is $b$. The height of the top wall, measured from the centerline is $h$, and is a function of both the longitudinal coordinate $x$ and the time $t$; the lateral coordinate is denoted as $y$. The corresponding velocities in the longitudinal and lateral directions are $u$ and $v$, respectively. The peristalsis of wavelength $\lambda$ travels in the $\mathrm{x}$-direction with speed $c$. The particle has a radius $R$ which is restricted to be less than $|a-b|$, allowing the particle to fit through the throat of the channel. If the particle were larger than the throat, it would be transported at the wave speed by virtue of its direct contact with the walls, and the problem would cease to be a fluid mechanics one.

The relevant independent variables are made dimensionless according to

$$
\xi=\frac{x}{\lambda} ; \quad \eta=\frac{y}{a} ; \quad \zeta=\frac{c t}{\lambda} ; \quad H=\frac{h(x, t)}{a} .
$$

Similarly, we can introduce several other dimensionless parameters, both geometrical and physical:

$$
\mathcal{V}=\frac{R}{a} ; \quad \operatorname{Re}=\frac{a c}{\nu} \alpha ; \quad \phi=\frac{b}{a} ; \quad \alpha=\frac{a}{\lambda} ; \quad \mathcal{D}=\frac{\rho_{s}}{\rho_{f}}
$$

Here $\mathcal{V}$ is a volume fraction; Re is the Reynolds number, with $\nu$ being the fluid kinematic viscosity; $\phi$ is the amplitude ratio; $\alpha$ is the normalized wavenumber; and $\mathcal{D}$ is the ratio of the solid to fluid density. Though many choices are availible, it was shown by Shapiro et al. [1] through an order of magnitude analysis that this is the appropriate Reynolds number, correctly describing the relative ratio of inertial to viscous forces, for the case where the inertial effects are small. Our simulations are performed in the regime of small, but finite, Reynolds number (no larger than $\mathcal{O}(10)$ ), and we maintain that this remains the appropriate definition for $R e$. 
To complete the specification of our problem, the wall shape $H$ takes the form

$$
H=1+\phi \cos 2 \pi(\xi-\zeta) \text {. }
$$

The wall motion is constrained to be in the lateral direction only, while the wave propogates longitudinally. The walls are extensible, meaning that they can readily undergo tangential extension to accomodate the prescribed motion [46]. Jaffrin et al. [10] claimed that "an inextensible wall would produce virtually the same flow except for very large values of $\alpha$ (i.e., short wavelengths)." In light of this comment, we restrict our simulations to small $\alpha$.

\section{Validation}

We will establish the veracity of our code in two steps. First we will compare the particlefree case with its analytical solution $[1,10,47]$. Then we will compare our results for particle transport with the Immersed Boundary Method (IBM) simulations of Fauci[23].

We first use our code to simulate the particle-free case, where there are known analytical solutions. The situation is the same as described in Section 3, except the particle has been removed. Velocity vectors and contours of pressure for a typical peristaltic flow are plotted in Fig. 4. In physical units, we set $a=0.05 \mathrm{~cm}$ and $c=0.8 \mathrm{~cm} / \mathrm{s}$, while the physical viscosity is adjusted to yield the desired $R e$ after $\alpha$ is specified; in corresponding lattice units $a=50$ and $\tau=0.75$. For this simulation $\phi=0.25, R e=0.5$, and $\alpha=0.25$, yielding $\nu=0.02 \mathrm{~cm}^{2} / \mathrm{s}$. Several features of the flow that were described in Section 1 can now be visualized, including two stagnation points on the channel centerline indicated by boxes marked $S 1$ and $S 2$ where the flow reverses direction. 


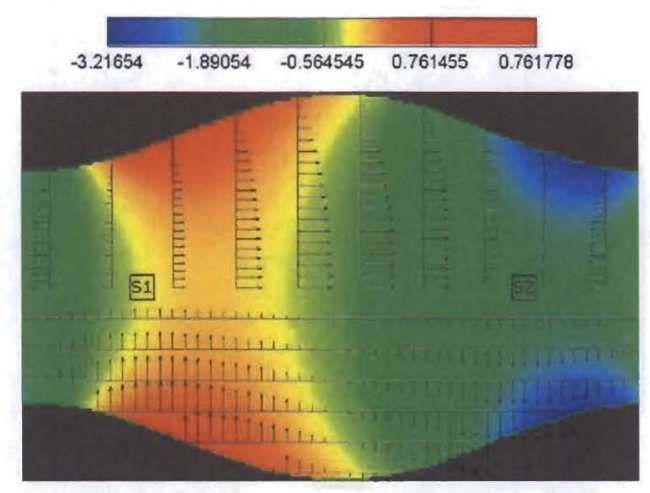

Figure 4: Velocity vectors over contours of pressure in a typical peristaltic flow without a particle. Vectors of $u$ are in the top half, and vectors of $v$ are in the bottom. Contours of pressure minus the pressure when there is no wall motion $\left(\left(p-p_{0}\right) /(\mu c / a)\right)$ are plotted normalized by a standard pressure normalization for low Reynolds number flows according to the colors of the scale.

A quantity of interest here is the time-mean volume flow rate $\bar{Q}$ calculated in the laboratory frame of reference. At a cross section $x=x_{0}$, the instantaneous volume flow rate is integrated over one full period of oscillation:

$$
\bar{Q}=\frac{1}{T} \int_{0}^{T} \int_{0}^{h} u\left(y, t ; x_{0}\right) \mathrm{d} y \mathrm{~d} t
$$

where $T=\lambda / c$ is the period and $x_{0}$ is arbitrary. The dimensionless mean flow is denoted $\Theta=\bar{Q} / a c$.

Shapiro et al. [1] and Jaffrin et al. [10] derived many results for the case where both $R e$ and $\alpha$ were negligible. If in addition there is no imposed pressure gradient(referred to as "free pumping" because there is no flow in the absence of wall motion), $\Theta$ can be given as a function of amplitude ratio:

$$
\Theta=\frac{3 \phi^{2}}{2+\phi^{2}}
$$




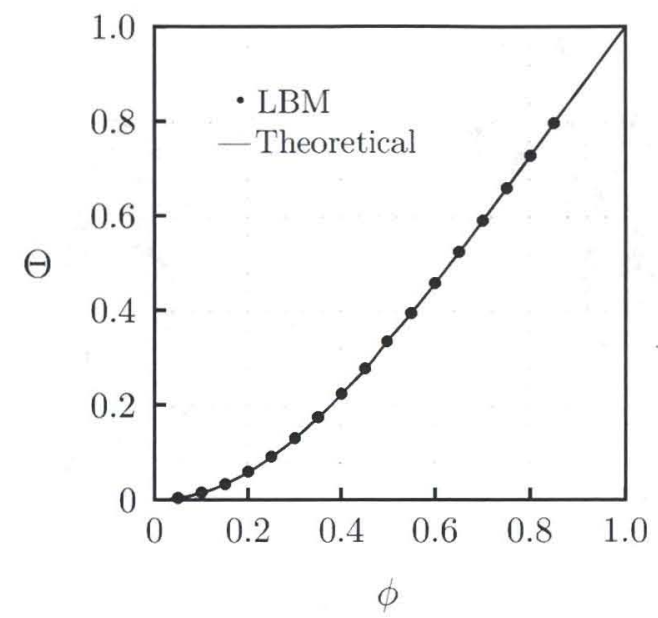

Figure 5: Time-mean volume flow rate as a function of amplitude ratio. $R e=0.5$, $\alpha=1 / 8 \pi$.

In Fig. 5, we compare our LBM results with the analytical solution where our geometric parameters are the same as in Fig. 4 except $\alpha=1 / 8 \pi, R e=1 / 4 \pi$, and $\tau=0.95$, using $\phi$ as the independent variable by changing $b$. Re and $\alpha$ should be chosen small for the simulation to be considered in the negligible inertia, infinite wavelength limit. Our simulation results agree with the analytical solution even at large amplitude ratios.

Unfortunately there are no known analytical solutions of peristaltic particle transport. However, we wish to demonstrate that our results compare favorably to the IBM simulations of Fauci[23], which to the best of our knowledge are the only other simulations of peristaltic transport of a finite particle. The simulation is specified by the parameters in Table 1, with both the fluid and the particle initially at rest, and the particle located on the centerline. In dimensional units, the positions and corresponding translational velocities of the center of mass of the particle are denoted $(\tilde{X}, \tilde{Y})$ and $(\tilde{U}, \tilde{V})$, respectively. Our comparison with Fauci is in physical units, but we state here their dimensionless analogs for future reference: 


$$
X=\frac{\tilde{X}}{\lambda} ; \quad Y=\frac{\tilde{Y}}{a} ; \quad U=\frac{\tilde{U}}{c} ; \quad V=\frac{\tilde{V}}{c} .
$$

\begin{tabular}{lccl} 
Domain Parameter & Symbol & Units & Value \\
\hline \hline Channel half-width & $a$ & $\mathrm{~cm}$ & 0.05 \\
Amplitude & $b$ & $\mathrm{~cm}$ & 0.008 \\
Wavelength & $\lambda$ & $\mathrm{cm}$ & 0.2 \\
Number of wavelengths & $N_{\lambda}$ & - & 1 \\
Wave speed & $c$ & $\mathrm{~cm} / \mathrm{s}$ & 0.8 \\
Kinematic viscosity & $\nu$ & $\mathrm{cm}^{2} / \mathrm{s}$ & 0.01 \\
Fluid density & $\rho_{f}$ & $\mathrm{~g} / \mathrm{cm}^{3}$ & 1.0 \\
\hline
\end{tabular}

\begin{tabular}{lccl} 
Particle Parameter & & & \\
\hline \hline Particle density & $\rho_{s}$ & $\mathrm{~g} / \mathrm{cm}^{3}$ & 1.0 \\
Particle radius & $\mathrm{R}$ & $\mathrm{cm}$ & 0.02 \\
Initial x-position & $\tilde{X}_{0}$ & $\mathrm{~cm}$ & 0.1 \\
Initial y-posision & $\tilde{Y}_{0}$ & $\mathrm{~cm}$ & 0.0 \\
Initial x-velocity & $\tilde{U}_{0}$ & $\mathrm{~cm} / \mathrm{s}$ & 0.0 \\
Initial y-velocity & $\tilde{V}_{0}$ & $\mathrm{~cm} / \mathrm{s}$ & 0.0 \\
\hline
\end{tabular}

Dimensionless Parameter

\begin{tabular}{lccl}
\hline \hline Wave number & $\alpha$ & - & 0.25 \\
Amplitude ratio & $\phi$ & - & 0.16 \\
Reynolds number & $R e$ & - & 1.0 \\
Volume fraction & $\mathcal{V}$ & - & 0.4 \\
Density ratio & $\mathcal{D}$ & - & 1.0 \\
\hline \multicolumn{4}{c}{} \\
LBM Parameter & \multicolumn{5}{l}{} \\
\hline \hline Relaxation time & $\tau$ & lattice-time & 0.95 \\
Grid spacing & $\Delta x / \delta x$ & cm/lattice-unit & 0.001 \\
Time step & $\Delta t / \delta t$ & s/lattice-time & $1.5 \times 10^{-5}$ \\
\hline
\end{tabular}

Table 1: Parameters of the comparison with Fauci simulation: The Base Case.

The LBM simulation is compared to the IBM result in Fig. 6 where the longitudinal velocity and position of the center of mass of the particle are plotted as a function of time. Since the particle was initially placed on the centerline of the channel, there was neither 
lateral migration nor rotation. Fauci's simulation data was not directly available, so Fig. 6 contains digitized reconstructions of the plots from her paper. It can be seen that while the LBM and the IBM simulations are not rigorously commensurate, the results are both qualitatively and quantitatively within reason. Therefore we proceed with subsequent simulations confident their output is genuine.

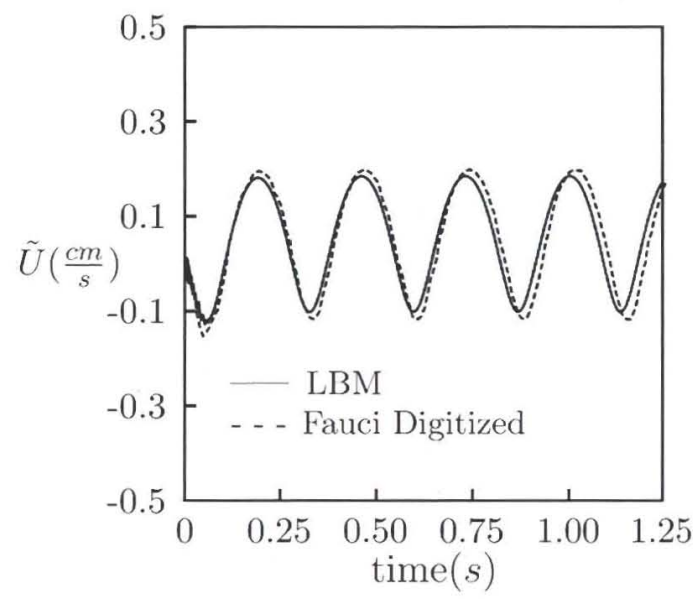

(a) Velocity

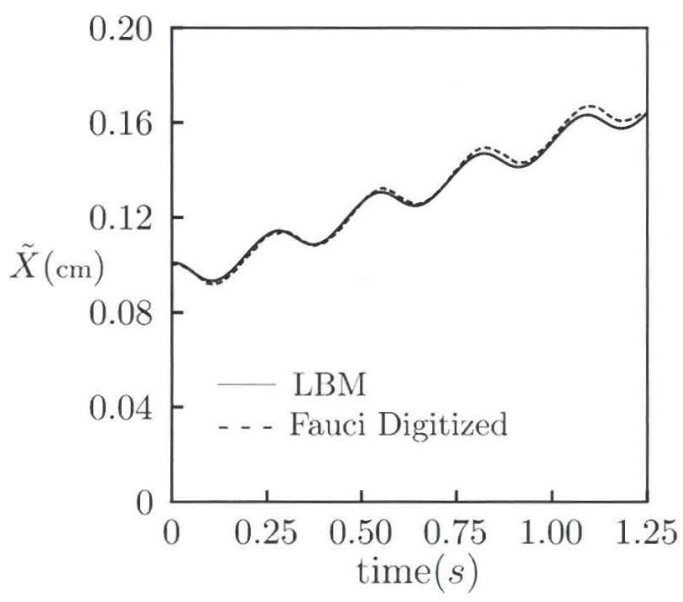

(b) Position

Figure 6: Longitudinal velocity and position of the center of mass of the particle as a function of time. -

\section{$5 \quad$ Variation of Nondimensional Parameters}

In this section we shall discuss some results pertaining to peristaltic particle transport. There are many parameters associated with this problem, and it would be impractical to study all possible combinations. We therefore establish a base case, namely the comparison with Fauci described in Section 4, from which we vary each of the relevant dimensionless parameters from eq. (12) independently to get a sense of its influence on the particle transport. For a particular group of simulations, the bulk of the parameters 
are those listed in Table 1, with the parameter of interest being varied.

The focus of this work is the transport of the finite-sized particle. Most of the simulations performed were of the nature shown in Fig. 6. Since lateral motion and rotation are mechanisms that decrease the longitudinal transport from its maximum value(discussed in Section 5.6), most of the simulations were performed with the particle initially located on the symmetric channel centerline. The particle developed an oscillatory character, translating back and forth in the longitudinal direction. After a short while, the motion became steady in a periodic sense, with a net displacement in the direction the wave was traveling. To get a sense of the averaged transport, the output parameter for the bulk of our simulations is the averaged longitudinal velocity of the particle

$$
\bar{U}=\frac{1}{c T_{p}} \int_{0}^{T_{p}} \tilde{U} \mathrm{~d} t
$$

where $T_{p}$ is the steady state particle period, not necessarily equal to the wave period, and normalized by the wave speed. Similar to the result described by Shapiro et al. [1] for a fluid particle, if a finite sized particle has a net positive displacement, it must travel for a time slightly greater than the wave period to find itself in the same position relative to the wave in order to begin the next particle period. This averaged velocity is found to be independent of the initial longitudinal position of the particle relative to the wave.

\subsection{Volume Fraction: $\mathcal{V}=R / a$}

The volume fraction is a measure of the size of the particle relative to the channel. $\mathcal{V}$ is designated the independent variable for this group of simulations, and is varied by changing the radius of the particle $R$. The other parameters are specified as in Table

1. Note the condition that $\mathcal{V} \leq 1-\phi$ for the particle to fit through the throat of 
the channel. The results for the simulations are displayed in Fig. 7. Also plotted are the results when the domain is extended to five and ten integral wavelengths, but still containing only the one particle. It is evident that the results are highly dependent on the length of the channel given the peiodic boundary conditions. The consequence of a finite domain is a departure from the assumption pervading the literature that the analysis of one wavelength is identical to any integral number of wavelengths. For a simulation where the domain is one wavelength, a periodic copy of the particle can be imagined to be one wavelength away, influencing the behavior of the actual particle. This influence diminishes as the size of the simulation is increased by virtue of the increased seperation between the particle and its periodic copy. This can be seen in Fig. 8 where the average transport of the base case is plotted for domains that are extended to include a larger integral number of wavelengths.

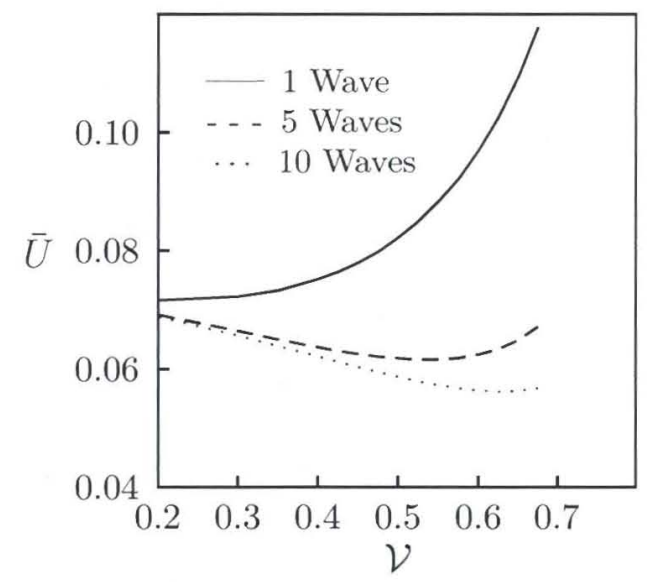

Figure 7: The average axial transport normalized by the wave speed as a function of volume fraction for different channel lengths. $R e=1.0, \alpha=0.25$, and $\epsilon=0.16$.

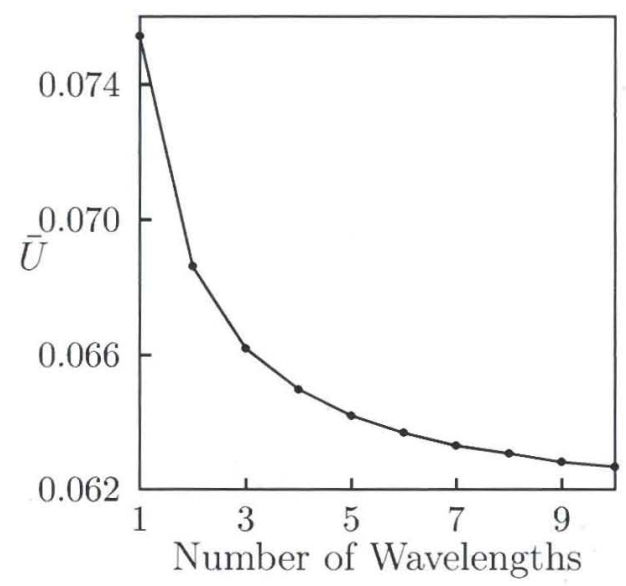

Figure 8: Longitudinal transport normalized by the wave speed as a function of integral number of wavelengths for the base case.

Fauci's simulations for varying the particle size produced a result similar to the onewavelength plot of Fig. 7, and she concluded that the transport increases with particle 
size. As evinced from the same figure, the transport may be artificially increased as a result of the periodic boundary, or some other factor which can mask the true influence of the particle size.

It is likely there are a number of factors that compete to produce the exhibited behavior. One factor that acts to decrease the transport as the particle size increases is the mass of the particle. An increased mass would require an increased force to accelerate it up to a given velocity. If this is the factor that dominates, one might expect to see the transport decrease with size as in the case with multiple wavelengths up to $\mathcal{V} \approx 0.5$.

Another factor to consider is the work done on the fluid-particle system by the peristaltic walls. The fact that velocity is prescribed on the walls can be misleading when making a conclusion about the work they perform on the system. In a physiological system, active muscular contraction combines with passive dilatation to produce the actual motion, where one can imagine a sensory feedback mechanism being in effect. The prescribed wall velocity boundary condition implicitly delivers the requisite surface forces to the system in order to achieve the desired wall motion. The mechanical energy equation, in the absence of applied body forces, can be stated:

$$
\frac{\partial}{\partial t}\left(\rho \frac{1}{2} v^{2}\right)+\nabla \cdot\left(\rho \boldsymbol{v} \frac{1}{2} v^{2}\right)=-\boldsymbol{v} \cdot \nabla p+\boldsymbol{v} \cdot(\nabla \cdot \boldsymbol{\tau})
$$

Here $\boldsymbol{\tau}$ is the viscous stress tensor(not to be confused with the LBM relaxation time). The first term represents the rate of increase of kinetic energy per unit volume, the second term represents the convection of kinetic energy, and the last two terms represent the increase of kinetic energy due to the scalar product of velocity with a surface force imbalance. One can imagine that the shear in the fluid is increased as the particle size increases. Since the wall velocity is prescribed, this increases the last term in eq. (18), 
thereby increasing the kinetic energy of the system, both fluid and particle, locally. This can be seen as a factor that increases the transport as the particle size increases.

Another factor to consider is the periodic boundary condition, which may be quantified by the ratio of the size of the particle relative to the length of the domain: $R / N_{\lambda} \lambda$. As we have mentioned above, the transport decreases with the length of the domain when the particle size is fixed, approaching some asymptotic limit. This suggests that the influence of the periodic copy on the particle can only be felt up to a finite distance away from the particle. When this is the case, $R / N_{\lambda} \lambda$ is small. The fact that the curves in Fig. 7 have a minimum for multiple wavelengths suggests that this influence of the periodic copy increases with particle size, as $R / N_{\lambda} \lambda$ increases for a fixed domain length.

The influence of the periodic copy on the actual particle can possibly be explained as follows. As the particle travels through the channel, it sheds a wake behind it. According to boundary layer theory for simple uniform flow past a bluff body, the defect velocity of the wake is proportional to the particle size, and it diminishes as $x^{-\frac{1}{2}}$. We are clearly not dealing with that idealized situation, but if there is a wake-like structure that is strong enough so that viscous effects cannot be considered negligible a distance on the order of the channel length behind the particle, the particle may interact with its own wake, and in a sense, draft itself; thereby increasing its transport. Evidence of this proposition was not found by analyzing the velocity in the system as motion induced from the peristaltic walls overshadowed any wake effects. However, Fig. 9 plots contours of velocity when the first order velocity calculated by simulating the same case without a particle has been subtracted away. The plot demonstrates that a second order wake-like structure stretches across the expanse of the domain for the case with the larger particle, while the same structure does not reach as far for the case with the smaller particle. It is possible that longitudinal transport of a particle is increased when this structure connects through the 
periodic boundary, even though it is most likely a second order effect.

Another possible explanation for why the transport decreases with channel length is as follows. As the particle size is increased, the increased shear leads to a local increase of kinetic energy caused by the peristaltic walls as was mentioned above. If the length of the domain is small, this increased kinetic energy is convected through the periodic boundaries just to enter the domain from the other side, keeping the kinetic energy throughout the domain at a relatively high level. If the domain is large, the increased kinetic energy in the vicinity of the particle may be convected into the relatively low kinetic energy regions away from the particle, thereby lowering the kinetic energy of the particle.

It is a complicated situation however, and the possibility of coupling effects among parameters makes it difficult to attribute this behavior to any single phenomenon. Most likely one of the previously mentioned factors will dominate the others for a particular situation. However, a variation in the particle size or domain length can cause a different factor to become important. The competition between these factors ultimately determines the transport.
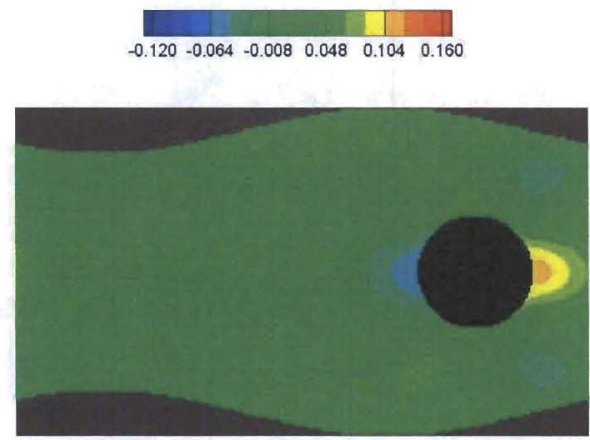
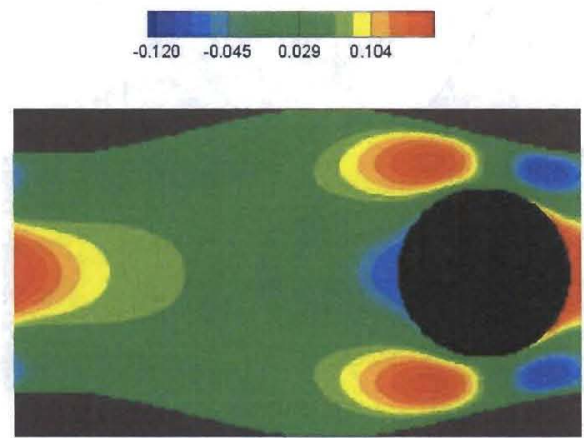

Figure 9: Comparison of wake-like structures behind particles of different sizes. The contours are colored by $u-u_{n p}$ in lattice units, where $u_{n p}$ is the velocity in the same domain and at the same time step when there is no transport particle. 


\subsection{Reynolds Number: $R e=(a c / \nu) \alpha$}

The Reynolds number is another interesting parameter to investigate. One may wonder if this is the best possible definition, because there are many options. While this definition was appropriate for the case without a particle, a different Reynolds number defined on the particle size might be more suited to characterize the situation. However, we have chosen to uphold the standard definition.

In our simulations, we vary the Reynolds number by changing the value of the physical kinematic viscosity. The result can be seen in Fig. 10a. The plot is nearly the same as that of Fauci, except this plot has been extended to $R e<1$, and it is in that range where the transport attains a maximum. The general consensus throughout the literature was that the effect of inertia was to decrease the transport. This assertion referred to the pumping capability in regards to volume flow rate in the absence of a particle, but it also transcends the context of particle transport, as seen in the figure.

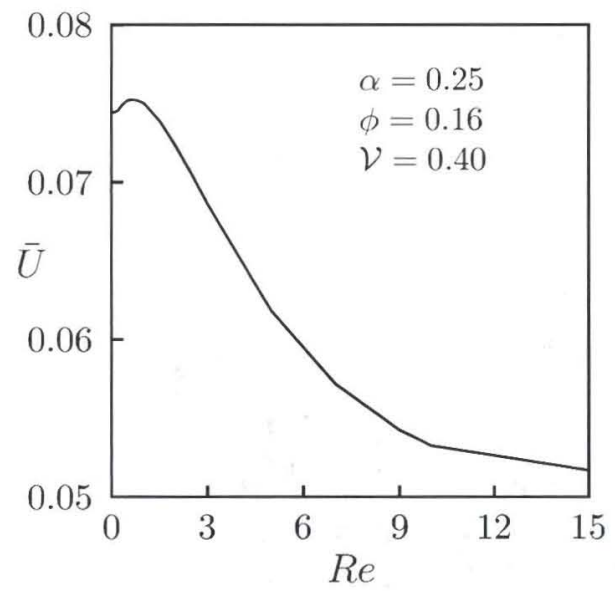

(a) Re with Max

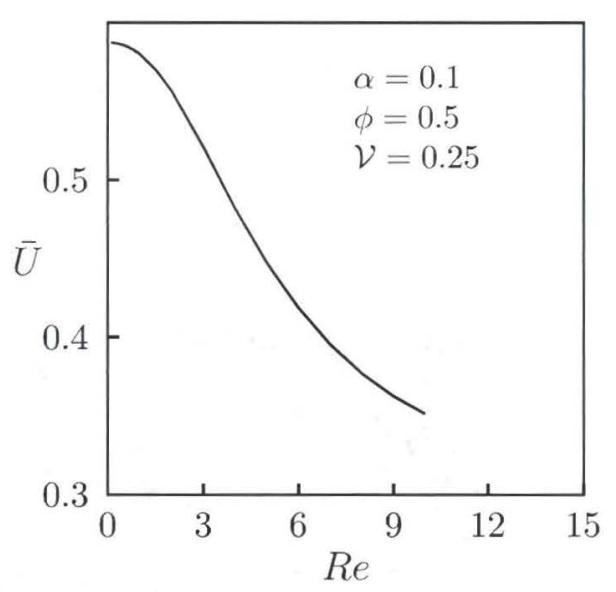

(b) Re with no Max

Figure 10: Longitudinal transport as a function of $R e$ under various conditions. (a) $\alpha=0.25$, $\phi=0.16, \mathcal{D}=0.4 ; \quad(b) \alpha=0.1, \phi=0.5, \mathcal{D}=0.25$.

For the most part, the transport decreases as Re increases, and approaches some 
minimum value. Under certain circumstances however, as in Fig. 10a, a slight amount of inertia can actually increase the transport. Jaffrin[47] expanded the standard particle free, negligible inertia equations by introducing $R e$ as a perurbation parameter. He similarly concluded that inertial effects can contribute favorably to the pumping performance under certain circumstances. Commensurate with the requirement of small Re for Jaffrin's analysis, the range where our particle achieves its maximum is conveniently observed only for $R e \leq 1$. In the plot, the maximum is achieved at $R e \approx 0.6$, where it levels off at an assymptotic value for smaller Re. The location of the maximum can be altered slightly by perturbing the simulation parameters by a small amount, but a drastic change collapses the maximum and the transport becomes a monotonically decreasing function of $R e$ as in Fig. 10b. To be more specific, if a maximum is achieved for a set of simulations with parameters $\left(\alpha_{m}, \phi_{m}, \mathcal{V}_{m}\right)$, then the set of simulations with parameters $\left(\alpha_{m}+\epsilon_{1}, \phi_{m}+\epsilon_{2}, \mathcal{V}_{m}+\epsilon_{3}\right)$ will most likely also mave a maximum, where $\left|\epsilon_{i}\right|$ represents a small perturbation. However, if the original parameters are altered by a significant amount, the set of simulations will most likely not contain a maximum. In the future, we would like to perform a set of simulations to compose a phase diagram that specifies what parameter combinations yield a maximum.

\subsection{Amplitude Ratio: $\phi=b / a$}

The amplitude ratio is a measure of the wall deflection relative to the width of the channel, and we vary $\phi$ in this group of simulations by varying the deformation amplitude $b$. For a given wave speed, the larger the deformation, the larger the lateral wall velocity must be to cover the increased distance in the same amout of time. This increases the shear which injects additional energy into the system as the deformation is increased similar to the 
way increased particle size also increased shear. The transport as a function of amplitude ratio is plotted in Fig. 11. It can be seen that the transport increases monotonically as a function of $\phi$. As has been mentioned throughout the literature, peristaltic pumping is much more effective at larger amplitude ratios.

It should be pointed out that the maximum allowable amplitude ratio for this particle size is $\phi=0.6$. However, large velocity gradients are developed as the particle passes through the throat of the channel as the amplitude approaches this maximum size. As a result, it was not possible to simulate cases where $|\phi+\mathcal{V}|$ was slightly less than unity, as the large velocity gradients led to a Mach number which exceeded the threshold for stable LBM simulations. If $1-\mathcal{V} \leq \phi \leq 1$, the simulation was stable, as the particle could not fit through the throat to cause the large velocity gradients, and the simulation was able to handle the particle-wall contact as described in Section 2.3. In this case, the particle was translated at the wave speed while contacting the wall.

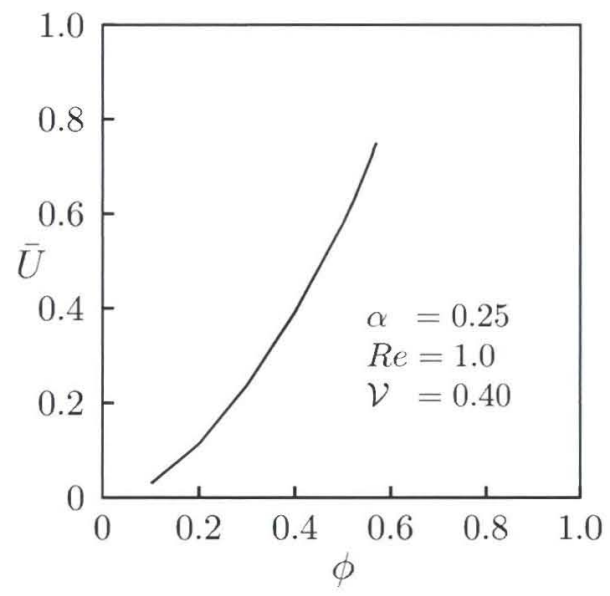

Figure 11: The average axial velocity normalized by the wave speed as a function of $\phi . R e=1.0, \alpha=0.25$, and $\mathcal{V}=0.4$.

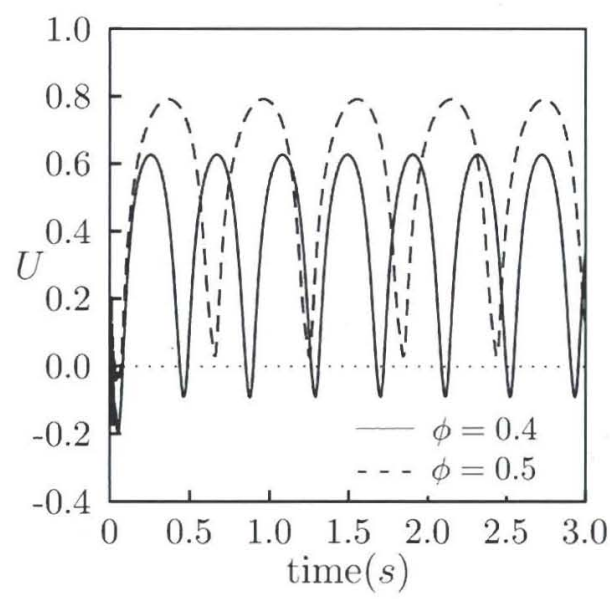

Figure 12: Instantaneous longitudinal velocity as a function of time for different values of $\phi .-\phi=0.4,--\phi=0.5$

One interesting phenomenon we have observed as the amplitude ratio is increased 
is that there is a critical point of transition, where the particle ceases to reverse its direction during the particle cycle. As can be seen in Fig. 12, the longitudinal velocity is still oscillatory, but there is a critical value of $\phi$ above which the instantaneous velocity of the particle is non-negative always. The critical amplitude ratio for this case is $\phi_{c}=0.48$.

\subsection{Nondimensional Wavenumber: $\alpha=a / \lambda$}

The dimensionless wavenumber is the ratio of the channel half-width to the wavelength. It was discussed earlier that many theoretical works assumed this to be a small parameter, but our simulations are not necessarily encumbered by those restrictions. For this set of simulations, we vary the dimensionless wavenumber by varying the wavelength. We have plotted our results in Fig. 13. The comments of Section 5.1 should be kept in mind as a change in $\lambda$ changes the length of the domain. The transport is seen to increase monotonically as $\alpha$ increases, and that the increase is linear beyond $\alpha \approx 0.3$. As was observed in Section 5.3, there is a critical point where the instantaneous velocity becomes non-negative only as $\alpha$ is increased, which is located at $\alpha=0.45$

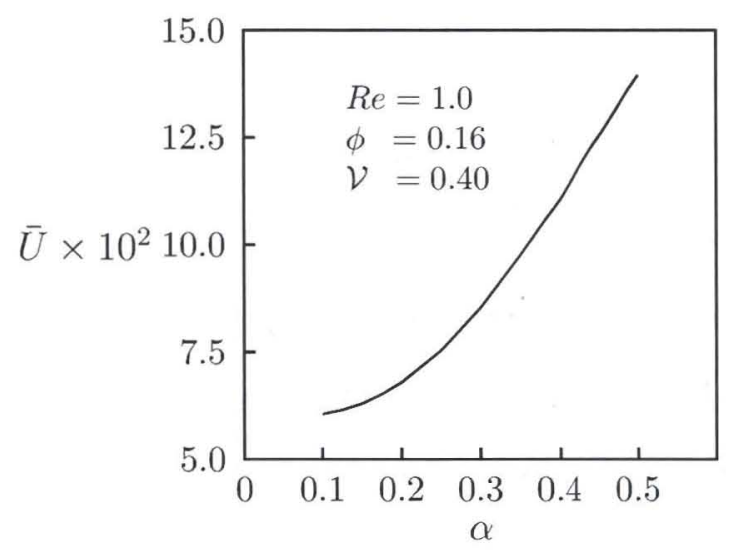

Figure 13: Average longitudinal velocity normalized by the wave speed as a function of $\alpha . R e=1.0, \phi=0.16$, and $\mathcal{V}=0.4$. 


\subsection{Density Ratio: $\mathcal{D}=\rho_{s} / \rho_{f}$}

Fig. 14a plots the transport as a function of density ratio without gravity by varying the solid particle density, with all other parameters fixed as in Table 1. As seen in Section 5.1 with the case of increasing particle size for long channels, an increase of the relative particle density causes a decrease in the transport, though not exactly linear. The trend is continued for particles that are less dense than the fluid as well. Fig. 14b also plots the transport as a function of density ratio, but in the presence of gravity directed opposite to the wave propogation. The ratio of gravitational to viscous forces can yield an estimate of the particle behavior in the presence of the body force. The gravitational force per unit length is approximated as $\left(\rho_{s}-\rho_{f}\right) g R^{2}$. The viscous term in the Navier-Stokes equations, $\mu \nabla^{2} \boldsymbol{u}$, can be appoximated as $\mu c / a^{2}$. Since this is a force per unit volume, we multiply by a term that scales as the mean area of the domain, $a \lambda$, to obtain a force per unit length. We also note that the resultant force on a macroscopic solid particle is typically an order of magnitude smaller than the individual forces as computed with the ME method. These forces can be combined and rearranged to give a modified Archimedes number:

$$
A r^{*}=\frac{\left(\frac{\rho_{s}}{\rho_{f}}-1\right) g R^{2} a}{0.1 \nu c \lambda}
$$

The modified Archimedes number for the base case with $\mathcal{D}=1.008$ is $A r^{*} \approx 1$. This tells us that the gravitational force is approximately equal and opposite to the viscous force induced by the peristalsis. Indeed this would seem to be an accurate characterization as Fig. 14b demonstrates that the particle has zero average longitudinal transport for $\mathcal{D}=1.008$. The transport in this case is also a decreasing function of the density ratio. Due to the fact that the variation in particle density is two orders of magnitude smaller than the density itself, we would not expect to see the same type of nonlinear behavior 
as in the gravity free case.

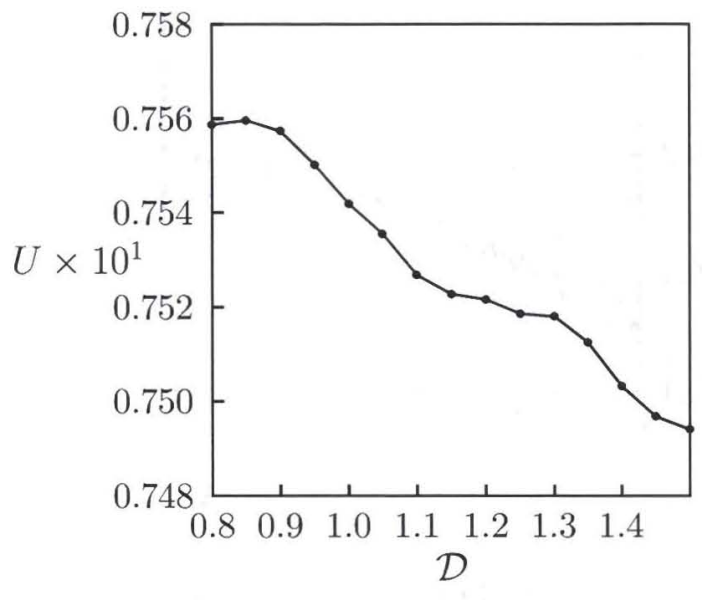

(a) No Gravity

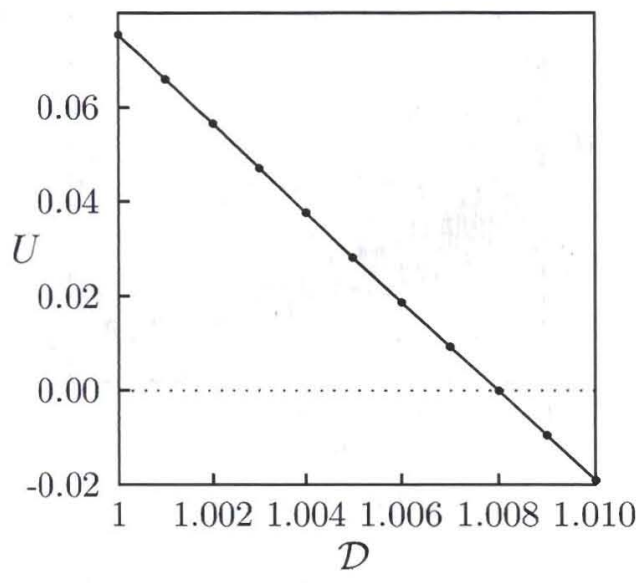

(b) Gravity in Longitudinal Direction

Figure 14: Longitudinal transport as a function of density ratio. $\alpha=0.25, \phi=0.16$, and $R e=1.0$. (a) $g_{x}=0 \mathrm{~m} / \mathrm{s}^{2} ;(b) g_{x}=9.81 \mathrm{~m} / \mathrm{s}^{2}$

\subsection{Lateral Position: $Y_{0}=\tilde{Y}_{0} / a$}

It has been seen that a particle which is initially placed on the symmetric centerline will remain there and will not rotate. If a particle is initially placed off the centerline, it will oscillate in both the lateral and angular directions as seen in Fig. 15. The parameters for these simulations are the same as in Table 1 except the initial lateral position has been changed to $Y_{0}=0.1$ and 0.2 , respectively. In an average sense, the particle migrates back toward the centerline, while the magnitude of the oscillation decreases. It is also observed that the oscillation is larger when the particle is initially placed at a larger lateral distance. The same trend is seen in the angular direction with the particle approaching some finite angle of rotation; the angle becomes larger when the initial lateral displacement is increased.

During the transient time while the offcenter particle approaches the centerline, the 


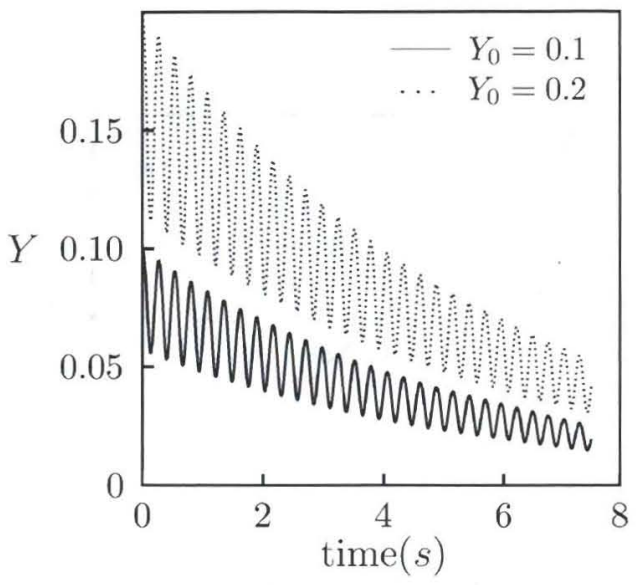

(a) Lateral Position

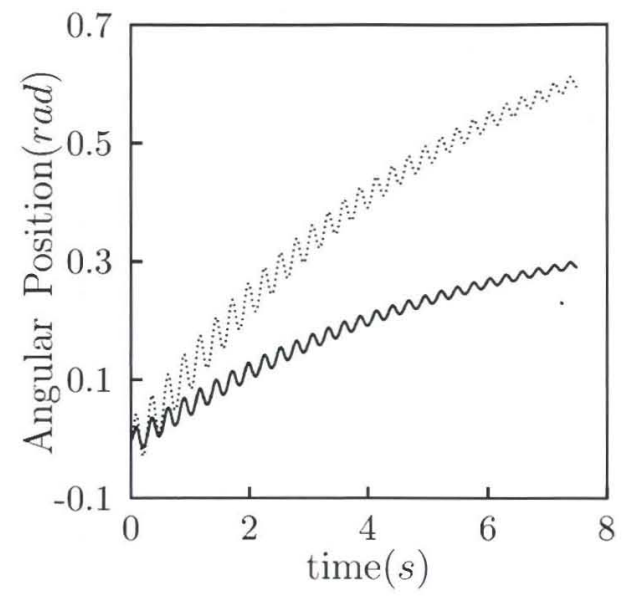

(b) Angular Position

Figure 15: Instantaneous lateral and angular positions as a function of time. $\alpha=0.25, \phi=0.16$, $\mathcal{D}=0.4$, and $R e=1.0$.

lateral translation and rotation cause a decrease in the longitudinal transport which would have otherwise been obtained by a particle that was symmetrically placed initially. It is assumed that these lateral and angular motions will damp out after some time as the particle settles at the stable configuration on the centerline; after which the particle would attain the same longitudinal transport as if it were initially placed on the centerline. This behavior can be seen in Fig. 16, as the average longitudinal velocity is plotted at each particle period for different initial lateral positions. This is in contrast to the observation made by Hung et al. [13] who found the particle to migrate slightly away from the axis. A possible explanation for this discrepency is that their experimental apparatus contained a single wave of deformation which acted only to increase the width of the channel from its unperturbed state, where our deformation wave produced sections that were both larger and smaller than the mean channel width. 


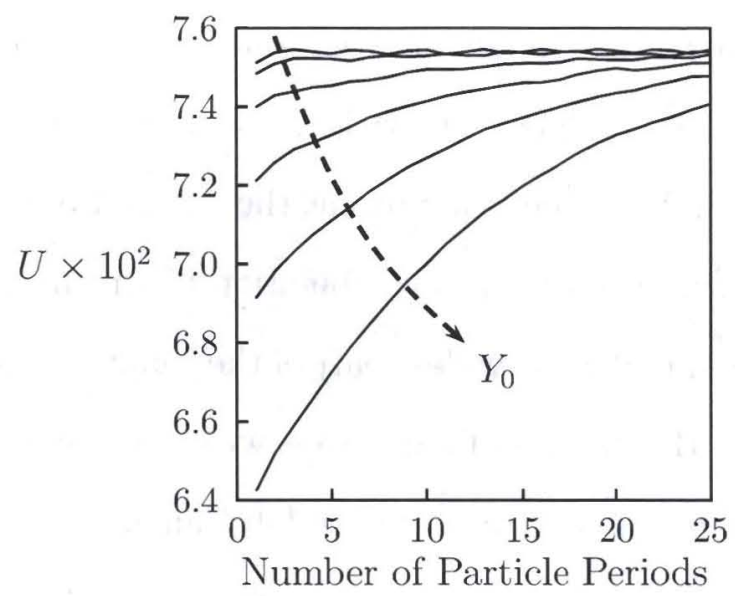

Figure 16: Average longitudinal velocity at each particle period for different initial lateral positions. $Y_{0}$ increases from 0.0 through 0.5 by increments of 0.1 in the direction indicated by the dashed line.

\section{Phenomenonalogical Results}

In the previous section, we have seen the influence of the dimensionless parameters on the transport of the particle. In this section, we will observe the behavior of the system under the peculiar condition of trapping. We will also analyze the effects of stress, pressure, and dissipation in the fluid.

\subsection{Trapping}

An interesting phenomenon related to peristaltic transport is "trapping[1]." Trapping occurs only in certain circumstances, typically characterized by a large amplitude ratio. When viewed in the wave frame of reference, a set of closed streamlines can be observed in the most dilated section of the channel. This set of streamlines represents a "bolus" of fluid that moves with the wave in the lab frame. There is an inner circulation present inside the bolus, but all the contained fluid particles move with a mean velocity equal to the wave speed. Fig. 17 exhibits an instance where trapping has occured without a particle. 
In this simulation, $\alpha=0.1$ and $\phi=0.75$; all other parameters are found in Table1 except the dimensional viscosity which is adjusted to yield $R e=1$. Streamlines near the walls adopt a shape similar to the walls as they circumvent the trapped bolus. The bolus itself consists of two counter-rotating vortices, the combination of which resembles an ellipse due to the wall shape, centered under the widest part of the channel. As these streamlines are closed in the wave frame, this bolus of fluid travels with the wave. This phenomenon cannot be witnessed by streamlines portrayed in the lab frame.

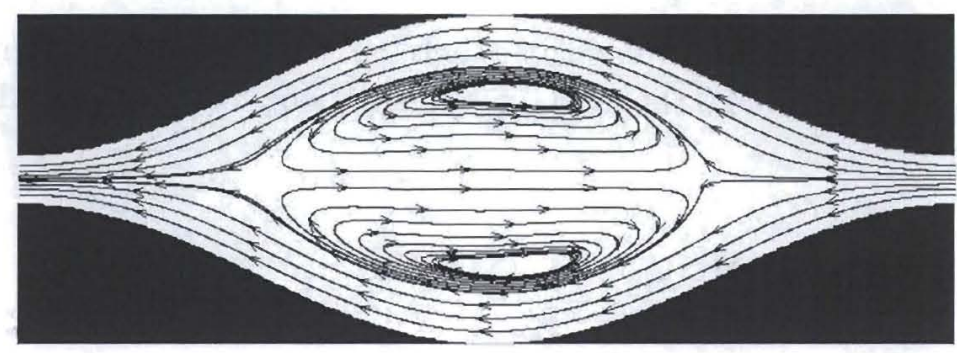

Figure 17: Trapping of fluid bolus.

Fig. 18 depicts what occurs when a particle is introduced to a situation where trapping occurs. The parameters are the same as in the particle-free trapping case, and the volume fraction $\mathcal{V}=0.2$. In the laboratory frame, both the wave and the particle move to the right, though the average velocity of the particle is less than the wave speed. Due to the fact that these snapshots are in the wave frame of reference, the particle progresses to the left in the pictures. The particle is initially located to the left of the bolus, where it passes through the throat of the channel between $t=0.208 \mathrm{~s}$ and $t=0.542 \mathrm{~s}$, crossing the periodic boundary on the left and entering again from the right. The particle arrives at the right of the bolus, and remains there for a long time as it is a neutrally stable location in the domain. Analogous to an inverted pendulum, the particle will not remain there if there is some perturbation in its lateral position. In this case, the particle is seen to 
enter the bolus, and follow the circuit specified by the streamlines. The presence of the particle inherently distorts the streamlines locally, but their general character remains intact. The particle does not escape the bolus, and develops a periodic circulation as if it were itself a fluid particle. As any other trapped fluid particle, the solid particle advances at the wave speed in the averaged sense, which is the maximum transport that is possible due to peristaltic effects exclusively. It would be advisable to transport particles under conditions where trapping occurs for this reason.

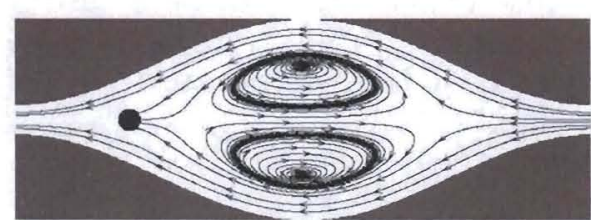

$\mathrm{t}=0.083 \mathrm{~s}$

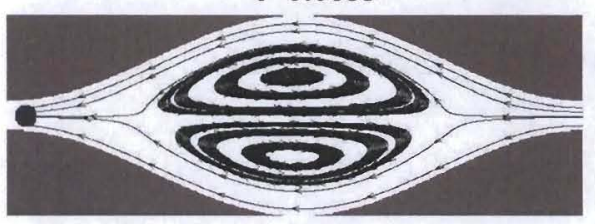

$\mathrm{t}=0.208 \mathrm{~s}$

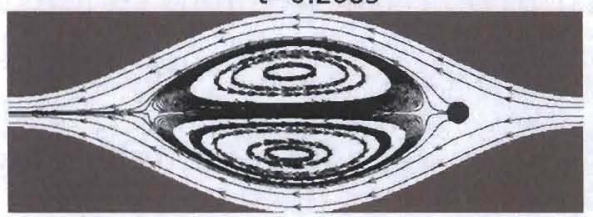

$\mathrm{t}=0.542 \mathrm{~s}$

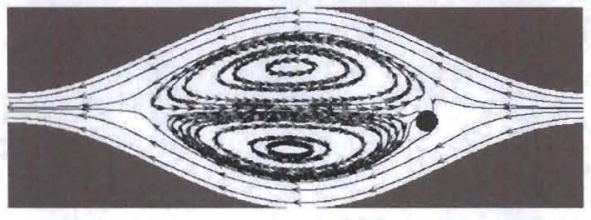

$t=1.667 \mathrm{~s}$

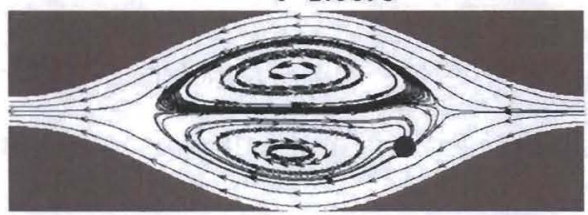

$\mathrm{t}=2.000 \mathrm{~s}$

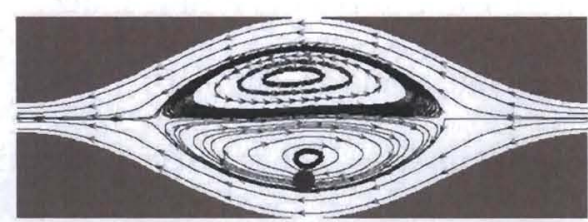

$\mathrm{t}=2.417 \mathrm{~s}$

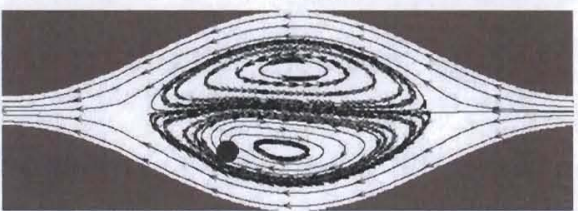

$\mathrm{t}=2.708 \mathrm{~s}$

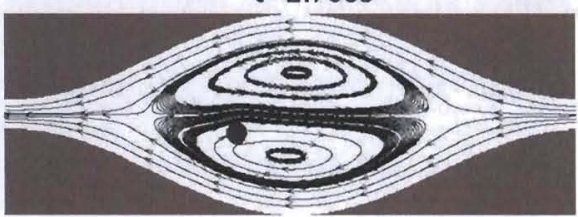

$\mathrm{t}=3.042 \mathrm{~s}$

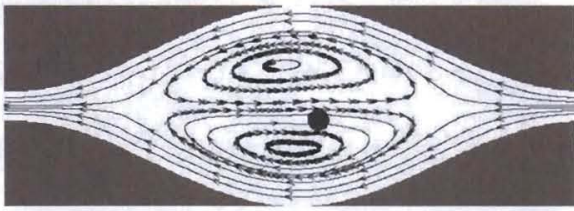

$\mathrm{t}=3.542 \mathrm{~s}$

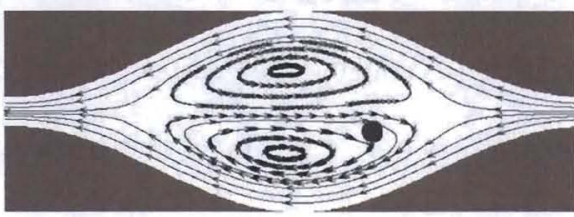

$\mathrm{t}=3.958 \mathrm{~s}$

Figure 18: Trapping of a solid particle in the bolus. 
Under certain circumstances, the particle may interact with the bolus in a different manner, depending on their associated properties. Ferrante et al. [48] studied two-way coupling in particle-laden tubulence, and in particular decomposed the particle-vortex interaction into several categories based on the relative timescales associated with the particle and vortex, respectively. As was described above, there are certain instances where the particle can enter the vortex and not ever subsequently possses sufficient inertia to eject itself. We have also witnessed the case where the particle does not have sufficient inertia to enter the bolus in the first place. We also expect there to be a case where the particle enters the bolus at the right, but is ejected at the left because its inertia does not allow it to trace the highly curved streamline.

\subsection{Viscous Stress, Pressure, and Dissipation}

It has been seen [49] that some particles which are often transported by peristalsis are shear-sensitive. For example, erythrocytes(red blood cells) are prone to hemolysis, the rupturing of the cell membrane and subsequent release of hemoglobin into the blood stream, when they are subjected to large shear stresses. Red blood cells have a larger tolerance for pressure and normal viscous stresses compared to shear stresses. In light of these circumstances, we can study the pressures and stresses in the vicinity of our macroscopic particle to gain some insight into the preferrable conditions for tansporting shear or otherwise sensitive particles.

In the LBM, pressure is calculated using an equation of state: $p=\rho c_{s}^{2}$. Here $c_{s}=1 / \sqrt{3}$ is the constant speed of sound. Inamuro [50] derived an efficient way to calculate the stress tensor, $\sigma_{i j}=-p \delta_{i j}+\tau_{i j}$, directly from the distribution functions, avoiding the need to take velocity gradients. For incompressible flow, the viscous or deviatoric stress tensor 
is defined as

$$
\tau_{i j}=2 \mu S_{i j} ; \quad S_{i j}=\frac{1}{2}\left(\frac{\partial u_{i}}{\partial x_{j}}+\frac{\partial u_{j}}{\partial x_{i}}\right)
$$

Here $S_{i j}$ is the symmetric strain-rate tensor and $\mu$ is the fluid dynamic viscosity. Inamuro's equation to calculate the full stress tensor is slightly modified to give the viscous stress tensor:

$$
\tau_{i j}=\frac{\tau-\frac{1}{2}}{\tau}\left(p \delta_{i j}-\sum_{\alpha} f_{\alpha}\left(e_{\alpha i}-u_{i}\right)\left(e_{\alpha j}-u_{j}\right)\right) .
$$

Here $i$ and $j$ are specified by $x$ and $y$, and $\delta_{i j}$ is the Kronecker delta symbol. The difference between $\tau$, the relaxation time, and $\tau_{i j}$ should be clear from the context.

Contours of normalized viscous stress and pressure are plotted in Figs. 19 and 20 as the particle passes critical locations in the channel for the base case. Notice that it is only necessary to plot one of the normal stresses, as $\tau_{x x}=-\tau_{y y}$ as a consequence of incompressibility; this statement was found to be accurate in our simulations. Relatively high shear stresses occur in the vicinity of the particle as it enters and leaves the throat region, though these stresses weaken during the instant the particle is centered in the throat. The shear stress on the particle is found to be a minimum when it is in the most dilated section of the channel. It would seem to be advisable to construct a peristaltic pumping apparatus for shear-sensitive particles where the intended particles are much smaller than the throat size, as the shear stress in that region is inversely proportional to the gap between the particle and the wall of the throat. There are seen to be two large regions of complimentary normal viscous stress. These regions alter their shape and enhance their magnitude as the particle passes through them. The normal viscous stress vanishes at the extreme lateral and longitudinal points along the particle surface. 
The particle experiences values of high and low pressure along its surface near its extreme lateral points as it enters the constricting section and leaves the diverging section of the channel respectively. Though the lateral pressures are seen to be extreme values, they are symmetric and consequently do not cause any lateral motion. The difference in pressures on opposite longitudinal sides seems to be small, but nevertheless influences the particle motion more than the extreme lateral pressures.
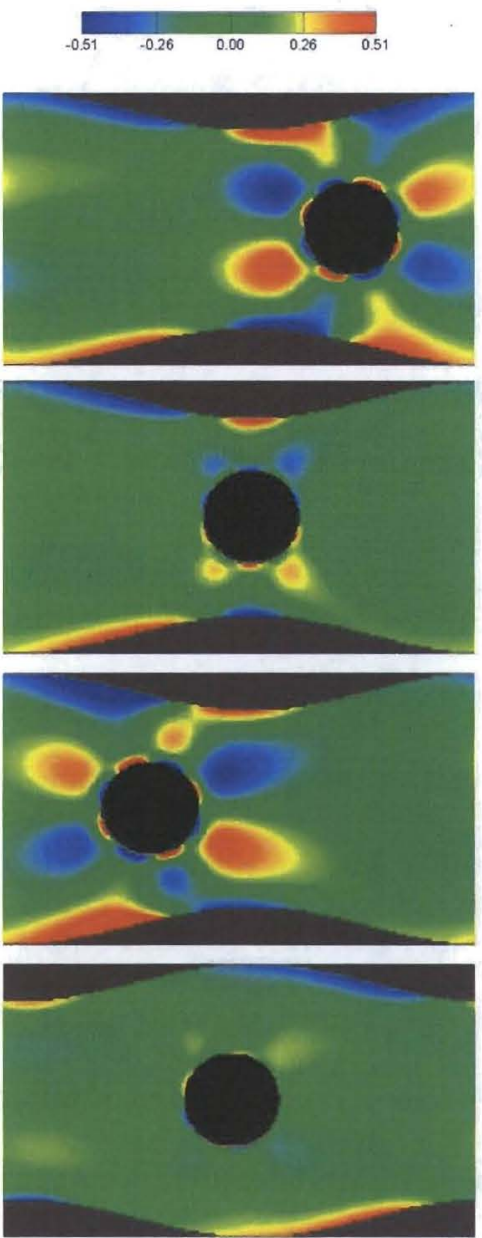

(a) Shear Stress: $\tau_{x y} /(\mu c / a)$
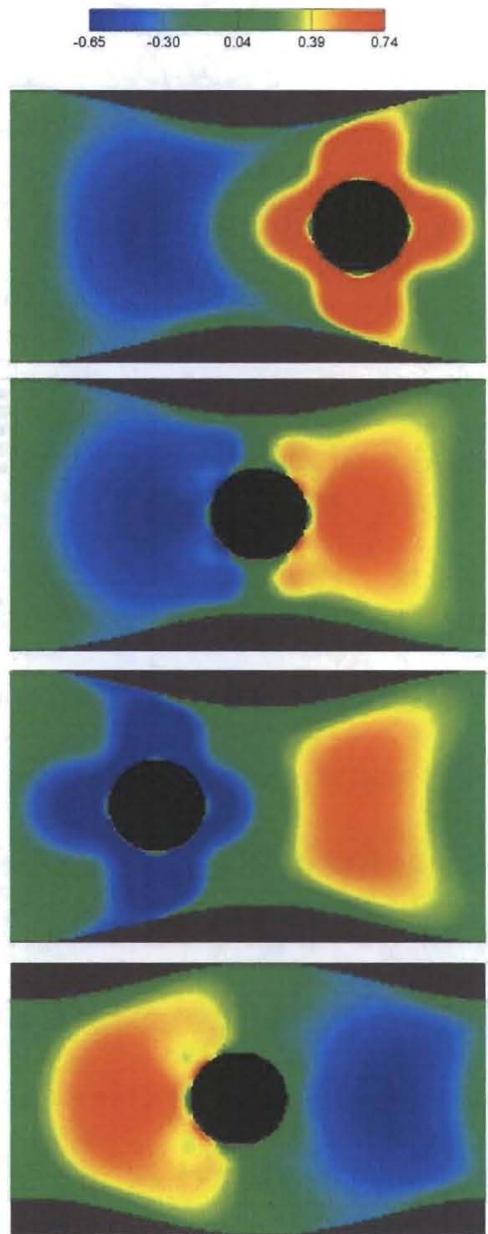

(b) Normal Stress: $\tau_{x x} /(\mu c / a)$

Figure 19: Contours of viscous stress normalized by $(\mu c / a)$ in the fluid at various particle locations for the base case. 

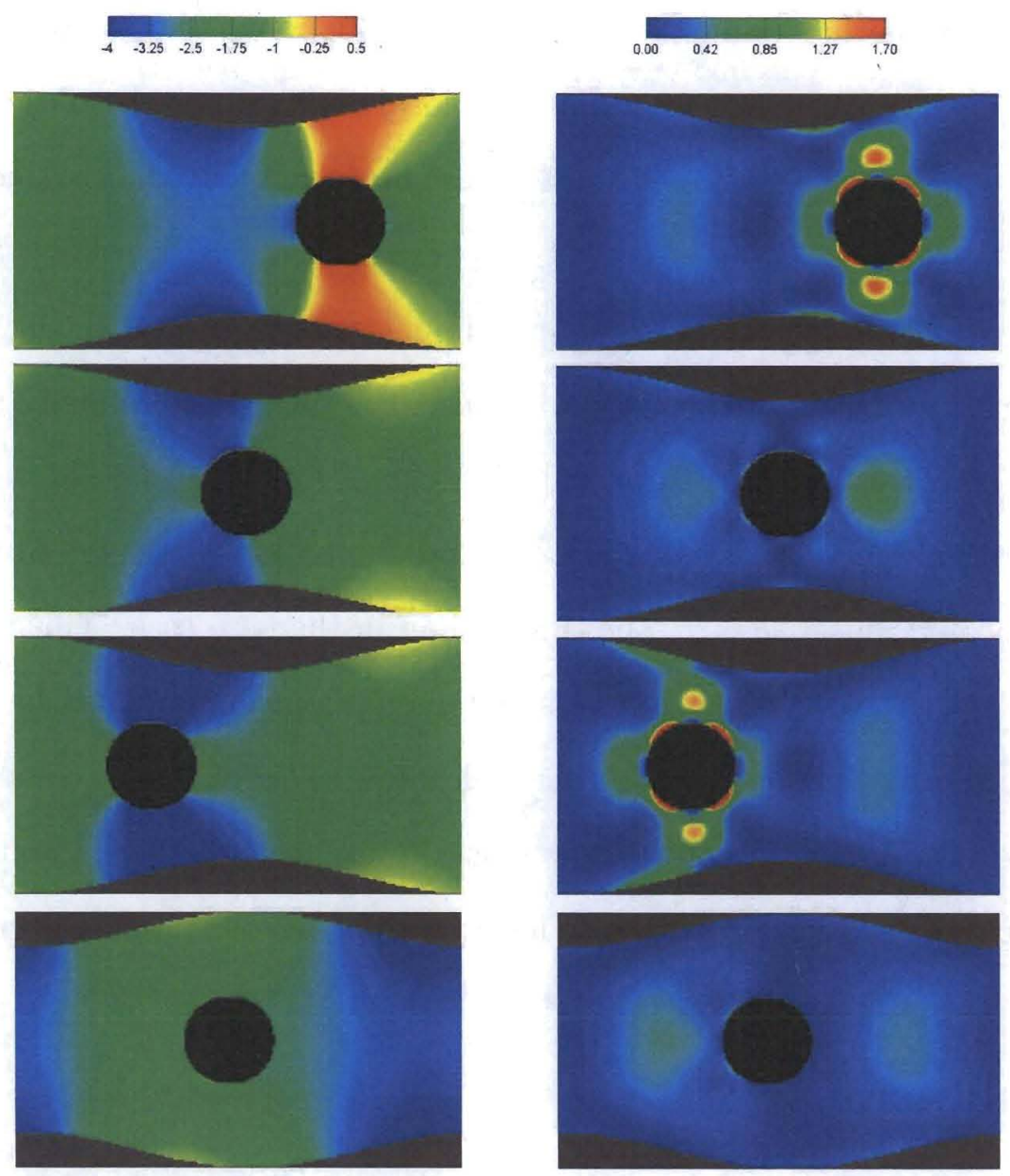

Figure 20: Contours of normalized pressure minus minus pressure when there is no wall motion $\left(\left(p-p_{0}\right) /(\mu c / a)\right)$ for the base case.

Figure 21: Contours of normalized dissipation $\left(\Phi /\left(\mu c^{2} / a^{2}\right)\right)$ in the fluid at various particle locations for the base case.

Another quantity of interest is the dissipation, $\Phi=\tau_{i j} S_{i j}$, which is the inner product of the viscous stress and strain-rate tensors and obtained by rearranging the last term of eq. (18). If the particle produces a large dissipation in the fluid, much of the kinetic energy produced from the walls is lost, which inevitably decreases the particle transport. As can be seen in Fig. 21, there are two regions of increased dissipation coinsiding with 
the locations of the lab frame centerline stagnation points that were pointed out in Fig. 4. As the particle enters these regions, the local dissipation is dramatically increased. Outside these regions however, the particle does not seem to have much effect on the total dissipation.

Contours of viscous stress, pressure, and dissipation can be seen in Fig. 22 where the particle is situated inside a trapped fluid bolus. The particle continually recirculates in this region, and does not pass through the throat of the channel. The region near the throat contains elevated levels of shear stress and dissipation, as the lab frame stagnation points are located much closer to the throat than in the base case. This trapped region is relatively inactive with regard to stress and dissipation compared to the throat region. As a result, the particle never experiences elevated levels of shear stress and does not increase the total dissipation of the flow much. This suggests that it would be prudent to transport shear-sensitive particles under conditions where trapping occurs.

\section{Conclusions}

The peristaltic transport of a macroscopic particle was simulated using the Lattice Boltzmann Method. The transport was systematically studied by gleaning the change in average longitudinal transport through variation of the associated dimensionless parameters. The average velocity was found to increase with particle size when the channel consisted of only one wave length, and was found to decrease with particle size when the channel consisted of five and ten integral wavelengths, only to increase again for the largest particle sizes. This suggested that the periodic boundary conditions influenced the system more than anticipated, and we have speculated that the increased transport is caused by the particle interacting with its own wake, and in a sense, drafting itself. The transport 

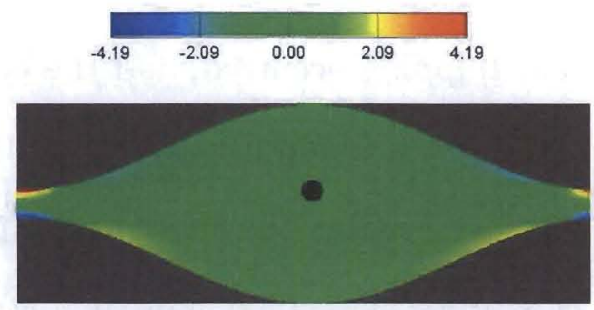

(a) Shear Stress: $\tau_{x y} /(\mu c / a)$
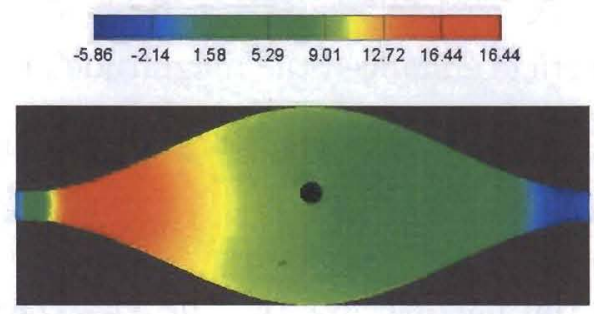

(c) Pressure: $\left(p-p_{0}\right) /(\mu c / a)$

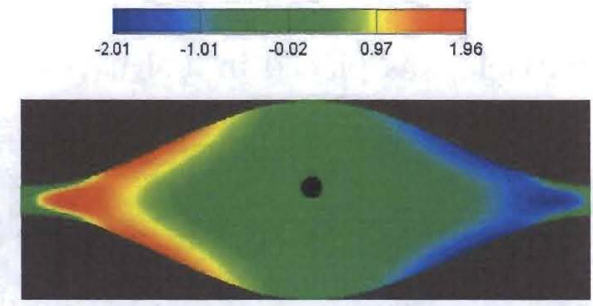

(b) Normal Stress: $\tau_{x x} /(\mu c / a)$

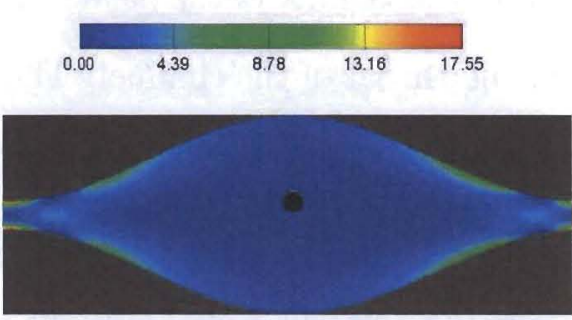

(d) Dissipation: $\Phi /\left(\mu c^{2} / a^{2}\right)$

Figure 22: Contours of normalized $(a)$ shear stress, $(b)$ normal viscous stress, $(c)$ pressure, and $(d)$ dissipation for the case when trapping occurs.

was found to decrease with Reynolds number, though some cases were found where the effect of a small amount of inertia can be to slightly increase the transport. The transport increased for increasing amplitude ratio, and a critical value of amplitude ratio was discovered where the particle no longer changes direction as it is transported forward. The transport increased for increasing dimensionless wavenumber, though the periodic boundary conditions may add a substantial contribution to this result as the channel length was varied. The transport decreased as the ratio of particle to fluid density was increased in the absence of gravity, but the decrease was not linear. The transport decreased linearly when gravity was directed opposite to the wave propogation, though the range of densities simulated was small, and nonlinear effects were not expected. A particle that was initially located off center was seen to exhibit both lateral and rotational oscillation. The oscillation amplitude damped out slowly as the particle eventually settled at the channel 
center.

The particle was placed in a situation where trapping occurred, and the particle was seen to enter the trapped bolus. Thereafter, the particle continually recirculated while following the streamlines viewed in the wave frame. Finally, the effect of the particle on values of stress, pressure, and dissipation in the fluid was studied. For the base case, the shear stress in the vicinity of the particle increased dramatically as the particle approached and exited the throat of the channel. The particle enhanced the magnitude and distorted the shape of two regions of complementary normal stress. The pressure was enhanced in the area between the side walls and the particle as the particle approached the throat, while it was decresed in the same area as the particle left the throat. Dissipation in the fluid was seen to increase locally when the particle was in the vicinity of the two stagnation points along the centerline where the flow changes direction when viewed in the lab frame. For the case where the particle circulated in the trapped bolus, the particle did not have much effect on stress, pressure, or dissipation, as it was confined to a region where fluid parameters had little fluctuation.

It is recommended to transport particles by peristalsis under the conditions of trapping. Firstly, a particle that circulates in a trapped fluid bolus is transported, on average, at the wave speed, which is the maximum possible transport in the absense of a favorable pressure gradient. Secondly, the particle does not experience elevated levels of shear stress along its surface while it is trapped, which is an important attribute when transporting shear-sensitive particles. Finally, the presence of the particle is not seen to increase the total dissipation in the fluid much compared to the case where the particle must pass through the throat, thereby most efficiently utilizing the energy transferred to the system by the peristaltic walls. 


\section{Acknowledgments}

This work is supported by Los Alamos National Laboratory's LDRD Program; Project

Number 20070267ER

\section{References}

[1] A.H. Shapiro, M.Y. Jaffrin, and S.L. Weinberg, "Peristaltic pumping with long wavelengths at low Reynolds number," J. Fluid Mech. vol. 37, part 4, 799 (1969).

[2] Y.C. Fung and C.S. Yih, "Peristaltic Transport," J. Applied Mech. vol. 35, no. 4, 669 (1968).

[3] C. Barton and S. Raynor, "Peristaltic Flow in Tubes," Bull. Mathematical Biophys. 30, 663, 1968.

[4] H.S. Maia and E.M. Coutinho, "Peristalsis and Antiperistalsis of the Human Fallopian Tube During the Menstrual Cycle," Biology of Reproduction 2, 305 (1970).

[5] M. Hanin, "The Flow Through a Channel Due to Transversally Oscillating Walls," Israel J. Tech. vol. 6, no. 1-2, 67 (1968).

[6] J.C. Burns and T. Parkes, "Peristaltic Motion," J. Fluid Mech. vol. 29, part 4, 731 (1967).

[7] T.F. Zien and S. Ostrach, "A Long Wave APproximation to Peristaltic Motion," J. Biomechanics 3, 63 (1970).

[8] P. Tong and D. Vawter, "An Analysis of Peristaltic Pumping," J. Applied Mech. vol. 39, no. 4, 857 (1972).

[9] P.S. Lykoudis and R. Roos, "The Fluid Mechanics of the Ureter from a Lubrication Theory Point of View," J. Fluid Mech vol. 43, part 4, 661 (1970).

[10] M.Y. Jaffrin and A.H. Shapiro, "Peristaltic Pumping," Ann. Rev. Fluid Mech. 3, 13 (1971).

[11] F.C.P. Yin and Y.C. Fung, "Comparison of Theory and Experiment in Peristaltic Transport," J. Fluid Mech. vol. 47, part 1, 93 (1971).

[12] S.L. Weinberg, E.C. Eckstein, and A.H. Shapiro, "An Experimental Study of Peristaltic Pumping," J. Fluid Mech. vol. 49, part 3, 461 (1971).

[13] T.K. Hung and T.D. Brown, "Solid-Particle Motion in Two-Dimensional Peristaltic Flows," J. Fluid Mech. vol. 73, part 1, 77 (1976).

[14] T.D. Brown and T.K. Hung, "Computational and Experimental Investigations of Two-Dimensional Nonlinear Peristaltic Flows," J. Fluid Mech. vol. 83, part 2, 249 (1977). 
[15] S. Takabatake and K. Ayukawa, "Numerical Strudy of Two-Dimensional Peristaltic Flows," J. Fluid Mech. 122, 439 (1982).

[16] C. Pozrikidis, "A Study of Peristaltic Flow," J. Fluid Mech. 180, 515 (1987).

[17] K.P. Selverov and H.A. Stone, "Peristaltically Driven ChannelFlows with Applications Toward Micromixing," Phys. Fluids vol. 13, no. 7, 1837 (2001).

[18] M. Yi, H.H. Bau, and H. Hu, "Peristaltically Induced Motion in a Closed Cavity with Two Vibrating Walls," Phys. Fluids vol. 14, no. 1, 184 (2002).

[19] V.P. Srivastava,and L.M. Srivastava, "Effects of Poiseuille Flow on Peristaltic Transport of a Particulate Suspension," Z. angew Math Phys. 46, 655 (1995).

[20] Kh. S. Mekheimer, E.F. El Shehawey, and A.M. Elaw, "Peristaltic Motion of a Particle-Fluid Suspension in a Planar Channel," Int. J. Theoret. Phys. vol. 37, no. 11, 2895 (1998).

[21] R. Usha, S. Senthilkumar, and E.G. Tulapurkara, "Numerical Study of Particulate Suspension Flow Through Wavy-Walled Channels," Int. J. Numer. Meth. Fluids 51, 235 (2006).

[22] M. Li and J.G. Brasseur, "Non-Steady Peristaltic Transport in Finite-Length Tubes," J. Fluid Mech. 248, 129 (1993).

[23] L.J. Fauci, "Peristaltic Pumping of Solid Particles," Computers Fluids vol. 21, no. 4, 583 (1992)

[24] C.K. Aidun and Y. Lu, "Lattice Boltzmann Simulation of Solid Particles Suspended in Fluid," J. Stat. Phys. vol. 81, nos. 1/2, 49 (1995).

[25] D. Yu, R. Mei, L.S. Luo, and W. Shyy, "Viscous Flow Computations with the Method of Lattice Boltzmann Equation," Progress in Aerospace Sciences 39, 329 (2003).

[26] A.J.C. Ladd, "Numerical Simulations of Particulate Suspensions via a Discretized Boltzmann Equation. Part 1.Theoretical Foundation," J. Fluid Mech. 271, 285 (1994).

[27] S. Chen \& G.D. Doolen, "Lattice Boltzmann Method for Fluid Flows," Annu. Rev. Fluid Mech. 30, 329 (1998).

[28] U. Frisch, D. d'Humières, B. Hasslacher, P. Lallemand, Y. Pomeau, and J.P. Rivet, "Lattice Gas Hydrodynamics in Two and Three Dimensions," Complex Systems 1, 649 (1987).

[29] G.R. McNamara \& G. Zanetti, "Use of the Boltzmann Equation to Simulate Lattice-Gas Automata," Physical Review Letters vol. 61, no. 20, 2332 (1988).

[30] H. Chen, S. Chen, and W.H. Matthaeus, "Recovery of the Navier-Stokes Equations using a LatticeGas Boltzmann Method," Phys. Rev. A vol. 45, no. 8, R5339 (1992).

[31] P.L. Bhatnagar, E.P. Gross, and M. Krook, "A Model For Collision Processes in Gases. I. Small Amplitude Processes in Charged and Neutral One-Component Systems," Physical Review vol. 94, no. 3, 511 (1954).

[32] Y.H. Qian, D. D'Humières, and P. Lallemand, "Lattice BGK Models for Navier-Stokes Equation," Europhys. Lett. vol. 17, no. 6, 479 (1992). 
[33] X. He \& L.S. Luo, "Lattice Boltzmann Model for the Incompressible Navier-Stokes Equation," J. Stat. Phys. vol. 88, no. 3/4, 927 (1997).

[34] A.J.C. Ladd, "Numerical Simulations of Particulate Suspensions via a Discretized Boltzmann Equation. Part 2.Numerical Results," J. Fluid Mech. 271, 311 (1994).

[35] O. Filippova and D. Hänel, "Lattice-Boltzmann Simulation of Gas-Particle Flow in Filters," Computers and Fluids vol. 26, no. 7, 697 (1997).

[36] R. Mei, L.S. Luo, and W. Shyy, "An Accurate Curved Boundary Treatment in the Lattice Boltzmann Method," J. Comput. Phys. 155, 307 (1999).

[37] C.K. Aidun, Y. Lu, and E.J. Ding, "Direct Analysis of Particulate Suspensions with Inertia using the discrete Boltzmann Equation," J. Fluid Mech. 373, 287 (1998).

[38] S. Chen, D. Martíncz, and R. Mei, "On Boundary Conditions in Lattice Boltzmann Methods," Phys. Fluids 8, 2527 (1996).

[39] H. Fang, Z. Wang, Z. Lin, and M. Liu, "Lattice Boltzmann Method for Simulating the Viscous Flow in Large Distensible Blood Vessels," Phys. Rev. E 65, 051925 (2002).

[40] R. Mei, D. Yu, W. Shyy, and L.S. Luo, "Force Evaluation in the Lattice Boltzmann Method Involving Curved Geornetry," Phys. Rev. E, 65, 041203 (2002).

[41] H. Li, H. Fang, Z. Lin, S. Xu, and S. Chen, "Lattice Boltzmann Simulation on Particle Suspensions in a Two-Dimensional Symmetric Stenotic Artery," Phys. Rev. E 69, 031919 (2004).

[42] H. Li, X. Lu, H. Fang, and Y. Qian, "Force Evaluations in Lattice Boltzmann Simulations with Moving Boundaries in Two Dimensions," Phys. Rev. E 70, 026701 (2004).

[43] M. Allen and D. Tildesley, "Computer Simulation of Liquids," Clarendon, Oxford (1987).

[44] R. Glowinski, T. Pan, T. Hesla, D. Joseph, and J. Periaux, "A Fictitious Domain Approach to the Direct Numerical Simulation of Incompressible Viscous Flow Past Moving Rigid Bodies: Application to Particulate Flow," J. Comput. Phys. 169, 363 (2001).

[45] Z. Feng and E. Michaelides, "The Immersed Boundary-Lattice Boltzmann Method for Solving FluidParticles Interaction Problems," J. Comput. Phys. 195, 602 (2004).

[46] G. Taylor; "Analysis of the Swimming of Microscopic Organisms," Proc. Roy. Soc. A 209, 447 (1951)

[47] M.Y. Jaffrin, "Inertia and Streamline Curvature Effects on Peristaltic Pumping," Int. J. Engng. Sci. 11, 681 (1973).

[48] A. Ferrante and S. Elghobashi, "On the Physical Mechanisms of Two-way Coupling in Particleladen Isotropic Turbulence," Phys. Fluids vol. 15, no. 2, 315 (2003)

[49] T.C. Hung, R.M. Hochmuth, J.H. Joist, and S.P. Sutera, "Shear-Induced Aggregation and Lysis of Platelets," Trans. Amer. Soc. Artif. Int. Organs 22, 285 (1976).

[50] T. Inamuro, K. Maeba, and F. Ogino, "Flow Between Parallel Walls Containing the Lines of Neutrally Buoyant Circular Cylinders," Int. J. Multiphase Flow 26, 1981 (2000). 